\title{
2 Educational Institutions in Information Science in Europe / Informationswissenschaftliche Ausbildungsinstitutionen in Europa / Institutions de formation pour les Sciences de l'Information en Europe
}

2.1 Institutions of Education / Ausbildungsinstitutionen / Institutions de formation

COUNTRY:

CITY, INSTITUTION:

ACADEMIC SUBJECT:

\author{
Austria \\ Graz, Karl-Franzens-Universität \\ Information Science (p. 141)
}

01 Karl-Franzens-Universitität Graz Institut für Informationswissenschaft Strassoldogasse 10 $8010 \mathrm{Graz}$ ÖSTERREICH

Tel: $\quad+43-316-3.80 .35 .60$

Fax: $\quad+43-316-38.14 .13$

E-mail: rauch@edvz.kfuni-graz.ac.at

02 Prof. Dr. Wolf Rauch (Leiter des Instituts, Tel: $+43(0) 316-3.80-35.60)$

031 Professor, 3 Universitätsassistenten, 1 nichtwissenschaftliche Mitarbeiterin

04 jährlich ca. 120 Studenten

05 1. Information Management: besondere Berücksichtigung des wirtschaftlichen Umgangs mit Information im betrieblichen Umfeld; 2. Information Engineering: Verbesserung der Informationsversorgung im Unternehmen, Datenmodellierung, Hypertext; $\underline{3}$. Information Assessment: Gesellschaftliche Auswirkungen

06 Das Institut für Informationswissenschaft der Karl-Franzens-Universität Graz ist in Österreich das erste und bisher einzige Universitätsinstitut auf diesem Fachgebiet. Durch seine Verankerung an der Sozial- und Wirtschaftswissenschaftlichen Fakultät bietet es vor allem Studenten der Betriebswirtschaftslehre im zweiten Studienabschnitt die Möglichkeit einer Ausbildung mit informationswissenschaftlichem Schwerpunkt. Auch für die Studenten der Volkswirtschaftslehre, Wirtschaftspädagogik und Soziologie sieht die Studienordnung die Gelegenheit einer informationswissenschaftlichen Ausbildung vor.

07 MA (Magister Artium) der Sozial- und Wirtschaftswissenschaften / Dr. der Sozial- und Wirtschaftswissenschaften (Informationswissenschaft) 
COUNTRY:

CITY, INSTITUTION:

ACADEMIC SUBJECT:
Austria

Wien, Österreichische Nationalbibliothek

Library and Information Science (p. 168)

01 Österreichische Nationalbibliothek Ausbildungsabteilung

Josefsplatz 1

1015 Wien

ÖSTERREICH

Tel: $\quad+43-1-53-4.10-3.57$

Fax: $\quad+43-1-53-4.10-3.58$

02 Dr. Gabriele Pum

03120 Dozenten und Lehrbeauftragte

04 ca. 200 Studenten

06 Informationsbroschüre: Grundausbildungslehrgänge Bibliotheks-, Dokumentations- und Informationswesen für die Verwendungsgruppe A Akademiker: Österreichische Nationalbibliothek. Information, Dokumentation, Bibliothek, Ausbildung. [o.J.]

07 Grundausbildungslehrgänge für die Verwendungsgruppen A+B-Bibliotheks-, Dokumentations- und Informationswesen (Verw. Gr. A. = Akademiker = Höherer Dienst, Verw. Gr. B. $=$ Maturanten $=$ Gehobener Dienst)

COUNTRY:

CITY, INSTITUTION:

ACADEMIC SUBJECT:

\section{Austria}

Wien, Wirtschaftsuniversität Wien

Information Management (p. 133)

01 Wirtschaftsuniversität Wien

Institut für Informationsverarbeitung und Informationswirtschaft

Abteilung für Angewandte Informatik insbesondere Betriebsinformatik

Augasse 2-6

1090 Wien

ÖSTERREICH

Tel: $\quad+43-1-3.30 .36 .36$

Fax: $\quad+43-1-3.30 .36 .36-10$

E-mail: kaiser@exaib.wu-wien.ac.at

02 Mag. Dr. Alexander Kaiser (Tel: +43(0)1-3.30.36.36.21) 
033 Professoren, 10 Assistenten, 10 Lektoren, 5 nicht-wissenschaftliches Personal

04 Etwa 30 Anfänger in der speziellen Betriebswirtschaftslehre je Semester.

05 Informationssysteme, Datenbanken, Information Retrieval, Rechtsinformatik

06 Studienführer der Besonderen Betriebswirtschaftslehre Informationswirtschaft (1993): 8., erweiterte und korrigierte Fassung. Abteilung für Angewandte Informatik insbesondere Betriebsinformatik. Institut für Informationsverarbeitung und Informationswirtschaft. Stand: 9. Juli 1993.

07 Magister der Sozial- und Wirtschaftswissenschaften (Mag. rer. soc. oec), Doktor der Sozial- und Wirtschaftswissenschaften (Dr. re. soc. oec) in Spezieller Betriebswirtschaftslehre Informationswirtschaft im Rahmen der Studien Betriebswirtschaft, Handelswissenschaft und Wirtschaftspädagogik an der Wirtschaftsuniversität Wien

COUNTRY:

CITY, INSTITUTION:

ACADEMIC SUBJECT:
Belgium

Antwerp, Universiteit Antwerp

Information and Library Science (p. 169)

01 Universiteit Antwerp / Université d'Anvers

Informatie- en Bibliotheekswetenschap / Information et bibliothéconomie

(Special Degree Information and Library Science)

Universiteitsplein 1

2610 Wilrijk-Antwerp

BELGIË

Tel: $\quad+32(0) 3-8.20 .28 .57$

Fax: $\quad+32(0) 3-8.20 .22 .47$

E-mail: desmet@pms.via.ac.be

02 Mr. Egbert de Smet

03 Coordination: 1 full-time scientific staff member; Administration: 1 part-time administration staff; part-time lecturers: 10, together equalling 1 full-time

05 Infometrics, Hypermedia Applications, Community Information Automation, Information Law, Interfaces, Electronic Documentary Systems

06 Interuniversity Special Degree. Information and Library Science. A two years postgraduate programme for information and library managers (1991): University of Antwerp, 2nd edition, March 1991

07 Information professionals in Library and Documentation (postgraduate degree). 
01 Université libre de Bruxelles

Faculté de Philosophie et Lettres

INFODOC-CP 142

avenue F. D. Roosevelt 50

1050 Bruxelles

BELGIQUE

Tel: $\quad+32(0) 2-6.50 .39 .43$

Fax: $\quad+32(0) 2-6.50 .39 .21$

02 Prof. Josiane Roelants (Course Leader) / Mrs. L. Lesoil (secretary)

03 Academic staff: 12 persons

0430 students

05 Computer linguistics; Training for non-academic female students

06 INFODOC. Sciences de l'Information et de la Documentation. Licence spéciale Certificat. Brochure d'Information (1991): Université Libre de Bruxelles, Mai 1991

07 Licence spéciale en Sciences de l'Information et de la Documentation; Doctorat en Philosophie et Lettres

! Licence en Science du Livre et de la Bibliothèque est possible.

Dès la fin de leurs études et grâce à la spécificité des cours offerts aux futurs étudiants et la réputation de qualité de la formation acquise, les diplômés de la section INFODOC sont pour la plupart engagés immédiatement dans la vie professionelle. 


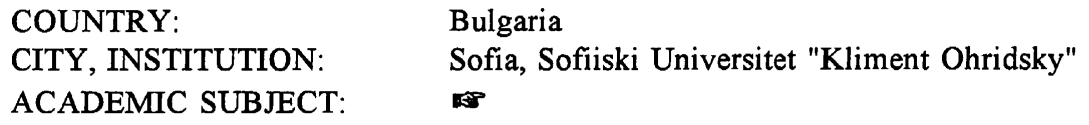

01 Sofiiski Universitet "Kliment Ohridsky"

Central Institute for Scientific and Technical Information (CISTI)

Boul. Ruski 15

1504-Sofia

BĂLGARIJA

Tel: $\quad+359(0) 56-84-19$

Telex: $\quad 23296$

! No more and detailed information having been received recently.

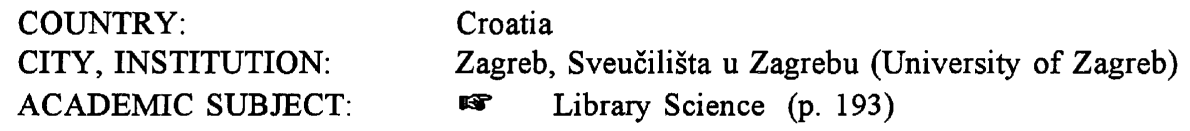

01 Sveučilišta u Zagrebu (University of Zagreb)

Filozofski fakultet Sveučilišta u Zagrebu (Faculty of Philosophy)

Odsjek za Informacijske Znanosti (Department of Information Sciences)

Dure Salaja 3

41000 Zagreb

HRVATSKA

Tel: $\quad+385-41-62.00 .06 / 2.17$

Fax: $\quad+385-41-51.38 .34$

02 Dr. Aleksandra Horvat (Dept. of Information Science, Chair of Librarianship)

031 professor, 2 associate professors, 1 assistant lecturer, other teachers from different departments of the faculty or different chairs or work in libraries

04 Librarianship: 25 students p.a.

Informatology-stream librarianship: 20 students p.a.

05 Theory of Librarianship; Theory of Information Science, Bibliographic Control and Management; History of the Book and Libraries

07 Diploma in Library Science / Informatology-Stream Librarianship 
COUNTRY:

CITY, INSTITUTION:

ACADEMIC SUBJECT:
Czech Republic

Praha, Univerzita Karlova

Information Science (p. 143)

01 Univerzita Karlova

Filozofická Fakulta

Katedra vědeckých informaci a knihovnictví

Celetná 20

11633 Praha

ČESKÉ ZEMĚ

Tel: $\quad+42(0) 2-22.84 .41$

Fax: $\quad+42(0) 2-2.35 .19 .11$

E-mail: len@csearn.bitnet

02 Prof. PhDr. Jiř́ Cejpek (Head of Department)

031 professor; 2 lecturers; 9 full-time senior lecturers; 1 scientific specialist; 2 nonscientific employees; 20 extra-mural specialists

04227 full-time and extra-mural students

05 Development, design and evaluation of information systems. Transforming of the latest results of information sciences into our curriculum.

07 Bachelor of Arts (BA), Master of Arts (MA), Doctor of Philosophy in Librarianship

COUNTRY:

CITY, INSTITUTION:

ACADEMIC SUBJECT:
Czech Republic

Praha, Vysoká Škola Ekonomická (University of Economics)

01 Vysoká Škola Ekonomická (University of Economics)

Department of Information and Knowledge Engineering

Nám W. Churchilla 4

13067 Praha

ČESKÉ ZEMĚ

Tel: $\quad+42-(0) 2-35.34 .28$

Fax: $\quad$ +42-(2)2-35.59.62

! No more detailed information has been received recently. 
COUNTRY:

CITY, INSTITUTION:

ACADEMIC SUBJECT:
Denmark

København, Danmarks Biblioteksskole

Library Science (p. 194)

Library and Information Science (p. 170)
01 Danmarks Biblioteksskole

The Royal School of Librarianship

6, Birketinget

2300 København $S$

DANMARK

Tel: $\quad+45(0) 31-58.60 .66$

Fax: $\quad+45(0) 32.84 .02 .01$
The Royal School of Librarianship Aalborg Branch 4 Langgagervej 9220 Aalborg DANMARK

$+45(0) 98-15.79 .22$

$+45(0) 98-15.10 .42$

02 Ole Harbo (Rector)

03 Teaching staff 46 (Copenhagen) and 17 (Aalborg). Total 63; non-teaching-staff: 68

05 The school runs 3-year research programmes that cover a rich diversity of Library and Information Science relevant themes.

07 Librarian in R.S.L. in Basic Library \& Information Studies Reseach Librarian in Research Librarianship Master in Library and Information Science (MLISc)

! The Royal School of Librarianship is an internationally recognized educational and research institution. Its activities are centred in two locations: a School in Copenhagen and a Department in Aalborg. The School is unique in several respects: it is a huge institution, probably one of the largest in the world, student enrollmens and faculty size are large and it boasts modern well-equipped accommodation with a wide range of resources and facilities for teaching and reseach. 
COUNTRY:

CITY, INSTITUTION:

ACADEMIC SUBJECT:
Estonia

Tallinn, Tallinn Pedagogical University

Library and Information Science (p. 170)

01 Tallinn Pedagogical University

Faculty of Library and Information Science

Department of Information Science and Bibliography

25 Narwa Road

EE 0010, Tallinn

EESTI

Tel: $\quad+(7) 01-42-42.23 .04$

Fax: $\quad+(7) 01-42-42.53 .39$

02 Silvi Roomets (Head of Department of Information Science and Bibliography)

033 Associate professors; 5 senior lecturers, 1 secretary

04180 full-time and 100 correspondence students

05 Information Science in the process of communication, establishment of computer-based data-bases in Estonia, information production, etc.

06 A Presentation 1992-1993 (1992): Tallinn Pedagogical University. Faculty of Library and Information Science. Tallinn 1992

07 University Diploma, Master's degree, Doctors degree in Librarianship and Information Science

COUNTRY:

Finland

CITY, INSTITUTION: $\quad$ Åbo/Turku, Åbo Akademi University

ACADEMIC SUBJECT: Library and Information Science (p. 171)

01 Åbo Akademi University

Department of Library and Information Science

Henriksgatan 9

20500 Åbo/Turku

SUOMI

Tel: $\quad+358(9) 21-65.43 .11$

Fax: $\quad+358(9) 21-65.45 .81$

E-mail: mignam@abo.fi / mlindquist@abo.fi

02 Prof. Mariam Ginman; Matts G. Lindquist (Acting Professor) 
031 Professor; 1 senior assistant; 1 lecturer; 1 research assistant; 1 part-time office clerk

04 Yearly intake of about 20 students.

05 Information needs; Collective development; Organizational studies; Information retrieval

06 Studies at Åbo Akademi University 1992 (1991): Information for foreign applicants. Åbo Akademi, International Office, 1991/1992

07 Masters degree, Licentiate, Doctorate in Library and Information Science

! The Language of Instruction is Swedish.

Studying at $\AA$ bo is free of charge, i.e., there is no institution fee. However, students do have to pay a compulsory fee (about FIM 460 during 1990) to the Students' Union. A bo cannot offer financial support to foreign students. Every year foreign students are required to prove to the Immigration Authorities that they have enough money to cover all expenses during stay in Finland.

The average living expenses of a single student is around FIM 3000 per month. Finding accomondation at a resonable price may be rather difficult in a university town, unless one gets a room or a flat in a student hotel.

COUNTRY

ACADEMIC SUBJECT:

Helsinki (Espoo), Teknillinen Korkeakoulu (Helsinki University of Technology)

叶

01 Teknillinen Korkeakoulu (Helsinki University of Technology)

Center for Continuing Education

02150 Espoo (Helsinki) 15

SUOMI

Tel: $\quad+358(9) 0-45.11$

Fax: $\quad+358(9) 0-451-40.68$

Telex: 125161

02 Merja Karivalo (Programm Leader)

! No more detailed information has been received recently. 
COUNTRY:

CITY, INSTITUTION:

ACADEMIC SUBJECT:
Finland

Tampere, Tampereen Yliopisto (University of Tampere)

Library and Information Science (p. 172)

01 Tampereen Yliopisto (University of Tampere)

Faculty of Social Sciences

Department of Information Studies

P.O. Box 607

33101 Tampere

SUOMI

Tel: $\quad+358(9) 31-15.61 .11$

Fax: $\quad+358(9) 31-15.65 .60$

E-mail: lirahu@uta.fi / lipeva@uta.fi

02 Prof. Pertti Vakkari

031 professor; 6 associate/assistant professors, 3 researcher, 1 assistant, 1 department secretary (all full-time)

04306 students

05 Information storage and retrieval; Information needs; Information resource management (IRM); Knowledge work

06 Annual Report 1992 (1993): University of Tampere: Departement of Information Studies 1993

International Guide for International Applicants. 1992-1993 Edition (1992): University of Tampere 1992

07 MSc (Master of Social Science), Licentiate of Social Science, Doctor of Social Science in Library and Information Science

! The Department is the leading research unit within Information Science in Finland. It produces about a half of the total national research output in Information Science. 


\section{COUNTRY: Finland \\ CITY, INSTITUTION: Vaasa, Vaasan Yliopisto (University of Vaasa) \\ ACADEMIC SUBJECT:}

01 Vaasan Yliopisto (University of Vaasa)

\section{POB 297}

65101 Vaasa

SUOMI

Tel: $\quad+358(9) 61-24.81 .11$

Fax: $\quad+358(9) 61-24.82 .08$

Telex: $\quad 74262$

E-Mail: thl@uwwasa.fi

02 Tuula H. Laaksovirta (Acting Professor)

! No more detailed information has been received recently.

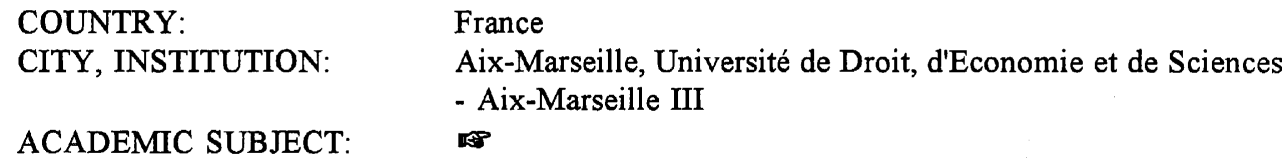

01 Université de Droit, d'Economie et de Sciences-Aix-Marseille III

Centre de Recherche Retrospective de Marseille (C.R.R.M.)

Centre scientifique de St. Jérôme

12 rue Ranque

13001 Marseille

FRANCE

Tel: $\quad+33-91.02 .90 .94$

Fax: $\quad+33-91.28 .80 .30$

Telex: $\quad 40.28 .76 .27 \mathrm{~F}$

02 Prof. Henri Dou

07 Licence avec mention Documentation

Maîtrise de Sciences de l'Information

Information Stratégique et Critique (DEA)

Diplôme d'Etudes Supérieures Spécialisées (DESS): Gestion des Systèmes Documentaires d'Information Scientifique et Technique

! No more detailed information has been received recently. 


\section{COUNTRY: $\quad$ France \\ CITY, INSTITUTION: Aix-Marseille, Université de Provence-Aix-Marseille I ACADEMIC SUBJECT:}

01 Université de Provence-Aix-Marseille I

94 blvd. Notre Dame

13006 Marseille

FRANCE

Tel: $\quad+33-91.95 .90 .71$

Fax: $\quad+33-91.50 .13 .00$

Telex: $\quad 40.20 .14$

02 Madame F. Chappaz

07 Diplôme de Documentation et Information Scientifiques et Techniques

! No more detailed information has been received recently.

COUNTRY:

CITY, INSTITUTION:

ACADEMIC SUBJECT:

\section{France}

Besançon, IUT de l'Université de Franche-Comté

Documentation (p. 105)

01 Institut Universitaires de Technologie (IUT)

de l'Université de Franche-Comté

Département Information-Communication

30 avenue de l'Observatoire

25402 Besançon Cedex

FRANCE

Tel:

$+33-95.46 .10 .45$

02 Madame G. Lallier (Directeur)

07 Diplôme Universitaire de Technologie (DUT) option Documentation d'Entreprise 


$\begin{array}{ll}\text { COUNTRY: } & \text { France } \\ \text { CITY, INSTITUTION: } & \text { Besançon, Université de Franche-Comté } \\ \text { ACADEMIC SUBJECT: } & \end{array}$

01 Université de Franche-Comté

UFR Sciences du Langage, de l'Homme et de la Société

30 rue Mégevand

25030 Besançon Cedex

FRANCE

Tel: $\quad+33-81.66 .54 .44$

02 Madame Marie-Madeleine Varet-Ingénieur

038 à 10 personnels scientifiques (enseignants et chercheurs/ingénieurs)

$04 \quad 14$ étudiants.

05 Bibliographie

06 DESS "Bibliographie Informatisée". Année Universitaire 1993-1994 (1993): Université de Franche-Comté Besançon 1993.

07 Diplôme d'Etudes Supérieures Spécialisées (DESS) en Bibliographie Informatisée

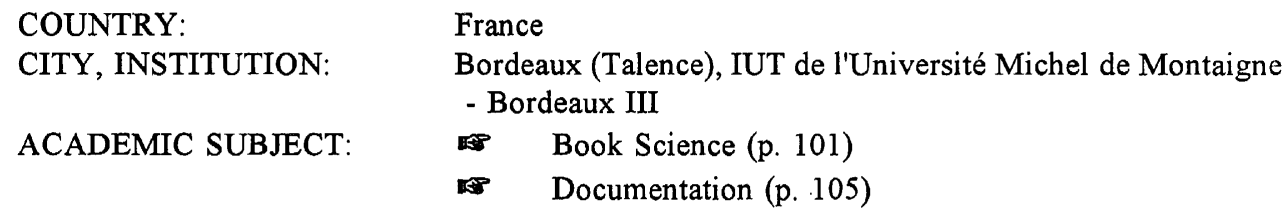

01 Institut Universitaire de Technologie (IUT) "B"

de l'Université Michel de Montaigne-Bordeaux III

Section Documentation d'entreprise

33405 Talence (Bordeaux) Cedex

FRANCE

Tel: $\quad+33-56.84 .44 .07$

Fax: $\quad+33-56.37 .06 .38$

02 Dr. Hubert Fondin

031 professeur; 1 maître de conferences; 1 prof. certificé en Sciences de l'Information; 7 enseignants de culture générale et nombreux volontaires professionnels 
10سm 14

04150 étudiants

05 Informatique documentaire; création des bases documentaires-téléréférence

07 Diplôme Universitaire de Technologie (DUT) option Documentation d'Entreprise Diplôme Universitaire de Technologie (DUT) option Métiers du Livre

COUNTRY: $\quad$ France

CITY, INSTITUTION: Bordeaux, Université Michel de Montaigne-

ACADEMIC SUBJECT: $\quad \begin{aligned} & \text { Bordeaux III } \\ & \text { Information Science (p. 144) }\end{aligned}$

01 Université Michel de Montaigne-Bordeaux III

UFR Lettres et Arts

Département Documentation

33405 Talence (Bordeaux) Cedex

FRANCE

Tel: $\quad+33-56.84 .50 .75$.

02 Dr. Hubert Fondin (Tel: $+33-56.84 .44 .07)$

031 professeur

$04 \quad 15-20$ étudiants

05 Informatique documentaire; Histoire en Sciences de l'Iinformation; Analyse d'objet

07 Maîtrise de Sciences de l'Information et de la Documentation 


$\begin{array}{ll}\text { COUNTRY: } & \text { France } \\ \text { CITY, INSTITUTION: } & \text { Dijon, IUT de l'Université de Bourgogne } \\ \text { ACADEMIC SUBJECT: } & \text { Book Science (p. 101) } \\ & \text { Documentation (p. 105) }\end{array}$

01 Institut Universitaire de Technologie (IUT)

de l'Université de Bourgogne

Département Information-Communication

Campus de Montmuzard, BP 510

21014 Dijon

FRANCE

Tel: $\quad+33-80.39 .50 .11$

Fax: $\quad+33-80.39 .50 .69$

Telex: $\quad 933-80667265$

02 M. M. Marty (Directeur)

07 Diplôme Universitaire de Technologie (DUT) option Documentation d'Entreprise Diplôme Universitaire de Technologie (DUT) option Métiers du Livre Licence avec mention Documentation Maîtrise de Sciences et Techniques: Certificat d'Attidude à la Documentation Automatisée

COUNTRY:
CITY, INSTITUTION:

ACADEMIC SUBJECT:

\section{France}

Grenoble, IUT de l'Université Pierre Mendès-FranceGrenoble II

Book Science (p. 101)

Documentation (p. 105)

01 Institut Universitaire de Technologie (IUT) de l'Université Pierre Mendès-France-Grenoble II Département Information-Communication Place Doyen Gosse 38031 Grenoble Cedex FRANCE

Tel: $\quad+33-76.82 .54 .00$

Fax: $\quad+33-76.82 .56 .54$

02 Madame S. Munoz (Directeur)

07 Diplôme Universitaire de Technologie (DUT) option Documentation d'Entreprise Diplôme Universitaire de Technologie (DUT) option Métiers du Livre 
血岗岗 16

Diplôme d'Etudes Approfondies (DEA) en Communication Homme-machine et Informatique Documentaire

COUNTRY:

CITY, INSTITUTION:

ACADEMIC SUBJECT:
France

Grenoble, Université Pierre Mendès-France-Grenoble II (CRISS)

01 Université Pierre Mendès-France-Grenoble II

Centre de Recherche en Informatique Appliquée aux Sciences Sociales (CRISS)

P.P. 47X

38040 Grenoble Cedex

FRANCE

02 Prof. J. Rouault

07 Diplôme d'Etudes Approfondies en Sciences de l'Information et de la Communication

! No more detailed information has been received recently.

$\begin{array}{ll}\text { COUNTRY: } & \text { France } \\ \text { CITY, INSTITUTION: } & \text { Le Havre, Université du Havre } \\ \text { ACADEMIC SUBJECT: } & \end{array}$

01 Université du Havre

25 rue Philippe Lebon, BP 1123

76063 Le Havre Cedex

FRANCE

Tel: $\quad+33-35.22 .92 .50$

Fax: $\quad+33-35.21 .49 .59$

07 Licence avec mention Documentation

! No more detailed information has been received recently. 


\author{
COUNTRY: $\quad$ France \\ CITY, INSTITUTION: Lyon (Villeurbanne), Ecole Nationale Supérieure des Sciences \\ ACADEMIC SUBJECT: \\ de l'Information et des Bibliothèques (ENSSIB)
}

01 Ecole Nationale Supérieure des Sciences de l'Information et des Bibliothèques (ENSSIB)

17/21 boulevard du 11 Novembre 1918

60623 Villeurbanne (Lyon) Cedex

FRANCE

Tel: $\quad+33(1) 78.89 .64 .45$

Fax: $\quad+33(1) 72.44 .27 .88$

02 Jacques Keriguy (Directeur)

07 Diplôme d'Etudes Supérieures Spécialisées (DESS) en Informatique Documentaire Systèmes Informationnels et Documentaires

Diplôme d'Etudes Approfondies (DEA) en Sciences de l'Information et de la Communication, option Information Scientifique et Technique

Diplôme d'Etudes Approfondies (DEA) en Sciences de l'Information et de la Communication, option Systémes d'Information Documentaire

Diplôme d'Etudes Approfondies (DEA) en Sciences de l'Information et de la Communication, option Systémes d'Information Stratégique

! No more detailed information has been received recently.
COUNTRY:
France
CITY, INSTITUTION:
Lyon (Villeurbanne), Université Claude Bernard-Lyon I
ACADEMIC SUBJECT:

01 Université Claude Bernard-Lyon I

Laboratoire d'Informatique Documentaire

43 boulevard du 11 Novembre 1918

69622 Villeurbanne (Lyon) Cedex

FRANCE

Tel: $\quad+33(1)-78.89 .81 .24$

Telex: $\quad 330208$

02 Madame Sylvie Laine

07 Diplôme d'Etudes Universitaires Scientifiques et Techniques (DEUST) Information et Documentation Scientifiques et Techniques

! No more detailed information has been received recently. 


\section{COUNTRY: $\quad$ France \\ CITY, INSTITUTION: Lyon, Université Lumière-Lyon II \\ ACADEMIC SUBJECT:}

01 Université Lumière-Lyon II

Centre de Recherche en Linguistique et Sémiologie

Av. Pierre Mendès France

86 rue Pasteur

69500 Bron (Lyon)

FRANCE

Tel: $\quad+33-78.69 .70 .00$

Fax: $\quad+33-78.69 .56 .01$

Telex: $\quad 33.06 .37$

02 M. Le Guerin

07 Licence avec mention Documentation

! No more detailed information has been received recently.

$\begin{array}{lll}\text { COUNTRY: } & \text { France } \\ \text { CITY, INSTITUTION: } & \text { Lyon, Université Jean Moulin-Lyon III } \\ \text { ACADEMIC SUBJECT: } & \text { Documentation (p. 106) } \\ & & \text { Information and Communication (p. 114-115) } \\ & \text { Information and Documentation (p. 121-122) }\end{array}$

01 Université Jean Moulin-Lyon III

Faculté des Lettres et Civilisations

Département Information, Communication et Documentation

74 rue Pasteur

69007 Lyon

FRANCE

Tel: $\quad+33-72.72 .20 .20 /+33-78.93 .74 .37$

Fax: $\quad+33-72.72 .20 .50 /+33-72.43 .96 .47$

Telex: $\quad 38.03 .11$

E-mail: catab@sun12.fr

02 Monsieur Bernard Wuilleme; Madam Jacqueline Rey (documentation); Madame Vidalenc-Sabourin (DEUST information-documentation)

039 enseignants titulaires, $1 / 2$ ingenieur de recherche 
04 Environ 160 étudiants p.a.

05 Nouvelle équipe ERSICO-ouverte sur les sciences cognitives et la linguistique.

07 Certificat Attidude Professionnelles Etudes Supérieures (CAPES) de Documentation Diplôme d'Etudes Universitaires Scientifiques et Techniques (DEUST) Information-Documentation

Maîtrise des Sciences de l'Information et de la Documentation

Licence, Maîtrise, Diplôme d'études supérieures spécialisés (DESS) d'Information et Communication

Diplôme d'Etudes Approfondies (DEA) en Sciences de l'Information et de la Communication en association avec l'Université Lumière Lyon II et l'Ecole Nationale Supérieure des Sciences de l'Information et des Bibliothèques

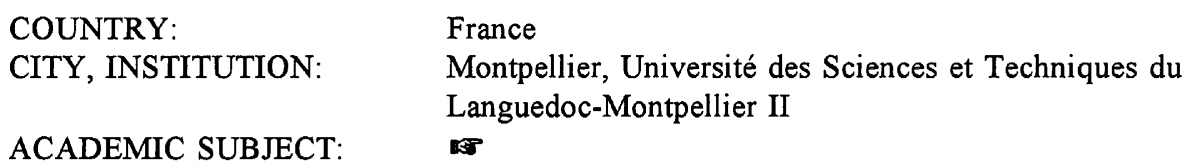

01 Université des Sciences et Techniques du Languedoc-Montpellier II

Place Eugène Bataillon

34095 Montpellier Cedex 5

\section{FRANCE}

Tel: $\quad+33-67.14 .30 .30$

Fax: $\quad+33-67.54 .30 .79$

Telex: $\quad 49.09 .44$

07 Licence avec mention Documentation

! No more detailed information has been received recently.

$\begin{array}{ll}\text { COUNTRY: } & \text { France } \\ \text { CITY, INSTITUTION: } & \text { Mulhouse, Université de Haute-Alsace } \\ \text { ACADEMIC SUBJECT: } & \end{array}$

01 Université de Haute-Alsace

Département Histoire et Métiers de la Culture

31 Grand Rue

68090 Mulhouse Cedex

FRANCE

02 J. P. Kintz (Directeur) 
07 Licence des Métiers de la Culture, des Archives et de la Documentation

! No more detailed information has been received recently.

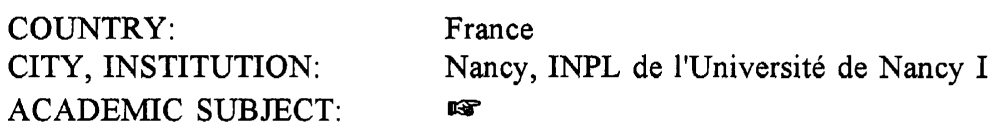

07 Diplôme d'Etudes Supérieures Spécialisées (DESS) en Information Scientifique et Technique

! No more detailed information has been received recently.

COUNTRY: $\quad$ France

CITY, INSTITUTION: $\quad$ Nancy, IUT de l'Université de Nancy II

ACADEMIC SUBJECT: Book Science (p. 101)

Documentation (p. 105)

01 Institut Universitaires de Technologie (IUT)

de l'Université de Nancy II

Département Information-Communication

2 bis Boulevard Charlemagne

54000 Nancy Cedex

FRANCE

Tel: $\quad+33-83.34 .46 .00 /+33-83.27 .30 .85$

Fax: $\quad+33-83.30 .05 .65$

02 Prof. Dr. Philippe Alexandre 
03 Personnel permanent (professeurs, maîtres de conférence, professeurs agrégés et certifiés), intervenants extérieurs venus du monde professionel. Le nombre des heures de présence est très variable.

04168 en première année; 40 en années spéciales

05 Informatique, Linguistique, Mass Media, Gestion et Traîtement de l'Information

07 Diplôme Universitaire de Technologie (DUT) option Documentation d'Entreprise Diplôme Universitaire de Technologie (DUT) option Carrières du Livre

COUNTRY:

CITY, INSTITUTION: ACADEMIC SUBJECT:
France

Paris, Conservatoire National des Arts et Métiers (CNAM)

Information Science (p. 145)

01 Conservatoire National des Arts et Métiers (CNAM)

Programme d'Information Scientifique et Technique

292 rue Saint-Martin

75141 Paris Cedex 03

\section{FRANCE}

Tel.: $\quad+33(1)-40.27 .23 .94$

Fax: $\quad$ +33(1)-42.71.93.29

E-mail: lecoadic@cnam.cnam.fr

02 Dr. Yves-François Le Coadic

03 personnel scientifique $=22(2$ plein-temps; 20 temps partiel $)$; personnel non-scientifique $=2$

0420 par année

05 usages et usagers/collaborations/cartes sémantiques

07 Diplôme d'Etudes Approfondies (DEA) en Information Scientifique et Technique 


$\begin{array}{ll}\text { COUNTRY: } & \text { France } \\ \text { CITY, INSTITUTION: } & \text { Paris, Ecole Nationale des Chartes } \\ \text { ACADEMIC SUBJECT: } & \text { Archive Science (p. 95) }\end{array}$

01 Ecole Nationale des Chartes

19 rue de la Sorbonne

75005 Paris

FRANCE

Tel: $\quad+33(1)-46.33 .41 .82$

Fax: $\quad+33(1)-43.29 .89 .75$

02 E. Poulle (directeur); Marie-Clotilde Hubert (secrétariat général)

03 Corps de neuf professeurs titulaires, deux maîtres de conférences, une dizaine de chargés de cours.

04 Nombre d'étudiants: 144

05 Toutes les disciplines relatives à l'histoire, à la civilisation et aux cultures de la France, des origines à l'époque contemporaine, et particulièrement à l'étude critique, à la mise en oeuvre, à la conservation et à la communication des documents qui s'y rattachent.

06 Notice sur l'Ecole des Chartes (1992): Paris, 19 rue de la Sorbonne, 1992

07 Diplôme d'archiviste paléographe

$\begin{array}{ll}\text { COUNTRY: } & \text { France } \\ \text { CITY, INSTITUTION: } & \text { Paris, Ecole de Bibliothécaires Documentalistes (EBD) } \\ \text { ACADEMIC SUBJECT: } & \end{array}$

01 Ecole de Bibliothécaires Documentalistes (EBD)

Institut Catholique de Paris

21 rue d'Assas

75270 Paris Cedex 06

FRANCE

Tel: $\quad+33(1)-42.22 .34 .52$

02 Madame Marie-Cécile Anfray (Directeur des études)

07 Diplôme de Bibliothécaire Documentaliste

! No more detailed information has been received recently. 
COUNTRY:

CITY, INSTITUTION:

ACADEMIC SUBJECT:
France

Paris, Institut d'Etudes Politiques de Paris (IEP)

Information and Documentation (p. 122)

01 Institut d' Etudes Politiques de Paris (IEP) /

Cycle supérieur de Spécialisation en Information et Documentation

5 rue de l'Abbaye

75006 Paris

FRANCE

Tel: $\quad+33(1)-44.07 .28 .63$

Fax: $\quad+33(1)-44.07 .28 .62$

02 Madame Martine Prevot-Hubert (Directeur des études, Tel: +33(1)-44.07.28.64)

031 directeur (professeur associé à l'Institut d'Etudes Politiques de Paris), 1 responsable des études (maître de conférence associé à l'Institut d'Etudes Politiques de Paris) + personnel enseignant à la fois universitaire et professionnel.

04 Matériels et bibliothèque pour les 20 étudiants en cours de scolarité

05 Formation à finalité professionnelle.

06 Cycle supérieur de Spécialisation en Information et Documentation. DESS 1993-1994 (1994): Institut d'Etudes Politiques de Paris. Fondation nationale des Sciences Politiques 1994

07 Diplôme d'Etudes Supérieures Spécialisées (DESS) en Information et Documentation

COUNTRY:

CITY, INSTITUTION:

ACADEMIC SUBJECT:
France

Paris, Institut National des Techniques de la Documentation (INTD-CNAM)

Information Management (p. 134)

Information Science (p. 146)

01 Institut National des Techniques de la Documentation (INTD)

Conservatoire National des Arts et Métiers (CNAM)

INTD-CNAM

2 rue Conté

75141 Paris Cedex 03

FRANCE

Tel: $\quad+33(1)-40.27 .25 .16 / 17$

Fax: $\quad+33(1)-48.87 .49 .37$ 
02 Bruno Delmas (Directeur de l'INTD); Mme Yasmina Abdellaoui (Tel: +33(1)40.27.26.73)

036 enseignants à temps plein; 30 enseignants à temps partiel

04 Nombre d'étudiants cycle diplôme technique: 100, cycle diplôme supérieur: 66; mastère spécialisé: 12

05 Veille technologique; Evalution des systèmes documentaires

06 Diplôme supérieur des Sciences et Techniques de l'Information et de la Documentation (1992): Paris INTD, 9/04/92 No. 613

Mastère spécialisé en Management de l'Information Stratégique 1992-1993 (1992): Paris INTD, Oct. 1992.

Spécialisation en Management de l'Information Stratégique (1992): Paris INTD, Juin 1992

07 Diplôme Technique de Documentaliste

Diplôme Supérieur des Sciences et Techniques de l'Information et de la Documentation Mastère Spécialisé en Management de l'Information Stratégique

! Ecole créée en 1950 au sein du CNAM pour les professionnels de l'information, l'INTD forme actuellement $20 \%$ des documentalistes en France, sur plusieurs niveaux de formation, que ce soit en formation initiale ou en formation continue. - Les salariés peuvent s'inscrire.

COUNTRY:

CITY, INSTITUTION:

ACADEMIC SUBJECT:

\author{
France \\ Paris, IUT de l'Université René Descartes-Paris V \\ Book Science (101) \\ Documentation (p. 105)
}

01 Institut Universitaire de Technologie (IUT)

de l' Université René Descartes-Paris V

Département Information-Communication

143 Ave de Versailles

75016 Paris

FRANCE

Tel: $\quad+33(1)-44.14 .45 .78$

Fax: $\quad+33(1)-44.14 .45 .73$

02 Madame Héléne Perennou-Soenen (Directeur)

035 enseignants-chercheurs d'Université ( 1 professeur, 4 maîtres de conférence): Ils doivent assurer 192 heures de cours par an 31 semaines. Deux sont responsables des tâches administratives d'organisation de la formation. Responsable de formation: 4 jours par 
semaine. Une trentaine de chargés de cours, enseignants extérieurs, experts dans le domaine qu'ils enseignent. 1 secrétaire.

0460 en moyenne, 80 avec les étudiants en Formation continue pour adultes lorsque ce groupe fonctionne.

06 Répertoire des Ecoles de Langue Française en Sciences de l'Information (1990): Réalisé par Héléne Soenen. Institut Universitaire de Technologie Université Paris V René Descartes. Association des Universités Partiellement ou Entièrement de Langue Française (AUPELF). Université des Réseaux d'Expression Française (UREF). Association Internationales des Ecoles des Sciences de l'Information (AIESI). 3ème Edition. Septembre 1990. S. 132-139

07 Diplôme Universitaire de Technologie (DUT): (fin de ler cycle universitaire) mention: Documentation d'Entreprise/Métiers du Livre

! Il existe en France une dizaine de départements d'IUT formant des documentalistes et des bibliothécaires. Documentalistes section Documentation d'entreprise au niveau ler cycle universitaire. Bibliothécaires section Métiers du livre.

$\begin{array}{ll}\text { COUNTRY: } & \text { France } \\ \text { CITY, INSTITUTION: } & \text { Paris, Université de Paris VII } \\ \text { ACADEMIC SUBJECT: } & \end{array}$

01 Université de Paris VII

Institut de Topologie et de

Dynamique des Systémes (ITODYS) U.P. VII

1 rue Guy de la Brosse

75005 Paris

FRANCE

02 Daniel Laurent (Directeur des études)

07 Diplôme d'Etudes Approfondies (DEA) d'Information Scientifique et Technique

! No more detailed information has been received recently. 


$\begin{array}{ll}\text { COUNTRY: } & \text { France } \\ \text { CITY, INSTITUTION: } & \text { Paris, Université de Paris VII } \\ \text { ACADEMIC SUBJECT: } & \end{array}$

01 Université de Paris VII

Unité fonctionnelle Cinéma, Communication, Information (UFCCI)

2 place Jussie

75251 Paris cedex 05

FRANCE

Tel: $\quad+33(1)-43.36 .25 .25$

02 Madame Denise Devèze-Berthet (Directeur des études)

07 Diplôme d'Etudes Approfondies (DEA) en Information Scientifique et Technique

! No more detailed information has been received recently.

COUNTRY:

France

CITY, INSTITUTION: Paris, Université de Vincennes à St. Denis-Paris VIII

ACADEMIC SUBJECT:

as

01 Université Vincennes à St. Denis-Paris VIII

Département Documentation

2 rue de la Liberté

93200 St. Denis (Paris)

FRANCE

Tel: $\quad+33(1)-48.21 .63 .64$

02 Prof. J.-P. Buffelan-Lanore

07 Maîtrise de Documentation et Information Scientifique et Technique

Maîtrise de Sciences de l'Information et de la Documentation

Maîtrise de Sciences et Technique: Diplôme de spécialisation, mention Documentation Maîtrise de Sciences et Techniques avec option Consacrée aux Techniques

! No more detailed information has been received recently. 


$\begin{array}{ll}\text { COUNTRY: } & \text { France } \\ \text { CITY, INSTITUTION: } & \text { Paris, IUT de l'Université de Paris X } \\ \text { ACADEMIC SUBJECT: } & \quad \text { Book Science (p. 101) }\end{array}$

01 Institut Universitaire Technologie (IUT)

de l'Université Paris-Nanterre-Paris X

200 Ave de la Republique

92001 Nanterre

FRANCE

Tel. $\quad+33(1) 40.97 .72 .00$

Fax: $\quad+33(1) 40.97 .75 .71$

07 Diplôme Universitaire de Technologie (DUT) option Métiers du Livre

COUNTRY:

CITY, INSTITUTION:

ACADEMIC SUBJECT:

\section{France}

Paris, Université Paris Nord-Paris XIII

Information Science (p. 146)

01 Université Paris Nord-Paris XIII

Av. Jean-Baptiste Clément

93430 Villetaneuse (Paris)

FRANCE

Tel: $\quad+33(1) 49.40 .30 .00$

Fax: $\quad+33(1) 49.40 .33 .33$

Telex: uninor $61.06 .70 \mathrm{~F}$

02 Madame Arlette Sancery (Responable des Relations Internationales, Tel: +33(1)49.40.37.65)

06 Guide de l'étudiant: Université Paris-Nord-XIII.

07 Licence, Maîtrise des Sciences de l'Information et de la Communication 
IIm.m.

$\begin{array}{ll}\text { COUNTRY: } & \text { France } \\ \text { CITY, INSTITUTION: } & \begin{array}{l}\text { Strasbourg, IUT de l'Université Robert Schuman- } \\ \text { Strasbourg III }\end{array} \\ \text { ACADEMIC SUBJECT: } & \text { Documentation (p. 105) }\end{array}$

01 Institut Universitaire de Technologie (IUT)

de l'Université Robert Schuman-Strasbourg III

Département Information-Communication

72 route du Rhin

67400 Illkirchen-Grafenstaden (Strasbourg)

FRANCE

Tel: $\quad+33-88.41 .42 .00$

Fax: $\quad+33-88.61 .30 .37$

02 P. Reinhold (Directeur)

07 Diplôme Universitaire de Technologie (DUT) option Documentation d'Entreprise Diplôme Universitaire de Technologie (DUT) option Documentation d'Entreprise en "année spéciale"

COUNTRY:

France

CITY, INSTITUTION:

Toulouse, Université Le Mirail-Toulouse II

ACADEMIC SUBJECT: Audiovisual Studies (p. 99)

01 Université Le Mirail-Toulouse II

Ecole Supérieure d'Audiovisuel

Institut Universitaire Professionnalisé

d'Ingéniérie de l'Information et de la Communication

5 allées Antonio Machado

31058 Toulouse Cedex

FRANCE

Tel: $\quad+33(1) 61.50 .44 .64$

Fax: $\quad+33(1) 61.50 .49 .34$

Service Minitel 3616 Code UTM-ESAV

02 Anne Marie Saboulard (Responsable Administratif, Tel. +33(1)61.50.44.51)

0318 enseignants titulaires et chargés de cours; 31 intervenants professionels

0436 DEUP, 40 Licence, 60 Maîtrise 
07 lére année DEUP d'Etudes Audiovisuelles

Licence d'Etudes Audiovisuelles

Maîtrise d'Etudes Audiovisuelles

COUNTRY:

CITY, INSTITUTION:

France

ACADEMIC SUBJECT:

Toulouse, IUT de l'Université Paul Sabatier-Toulouse III

Documentation (p. 105)

01 Institut Universitaire de Technologie (IUT)

de l'Université Paul Sabatier-Toulouse III

Département Information-Communication

115 route de Narbonne

31077 Toulouse Cedex

FRANCE

Tel: $\quad+33-62.25 .81 .80$

Fax: $\quad+33-62.25 .81 .01$

Telex: $\quad 52.18 .80$

02 Madame Daniele Cambrezy

037 enseignants de statut 2ème degré (langues vivantes, expression, histoire etc.): $384 \mathrm{~h}$ d'enseignement par an minimum. Pas d'obligations de recherche. 9 enseignants-chercheurs (1 professeur, 6 maîtres de conférence, 2 ATER): 192 h d'enseignement minimum + recherche. 3 PAST (professionels associés, sur poste pour 3 ans): $192 \mathrm{~h}$ enseignement + recherche. Vocataires professionels.

04 Nombre d'étudiants: 160

05 Recherche théorique et appliquée dans le champ des Sciences Sociales et Humaines dans le cadre du LERASS, laboratoire de l'IUT.

07 Diplôme Universitaire de Technologie (DUT) option Documentation d'Entreprise

! Nous avons un projet de création d'une option "Communication d'Entreprise" de l'IUP de Toulouse et d'une Année Spéciale en Communication. L'un des groupes de Documentation est une formation à orientation "trilingue" (anglais et allemand ou espagnol). 


\section{COUNTRY: $\quad$ France \\ CITY, INSTITUTION: Tours, IUT de l'Université de François Rabelais \\ ACADEMIC SUBJECT: Documentation (p. 105)}

01 Institut Universitaires de Technologie (IUT)

de l'Université de François Rabelais

Département Information-Communication

29 rue du Pont Volant

37023 Tours Cedex

FRANCE

Tel: $\quad+33-47.36 .66 .00$

Fax: $\quad+33-47.36 .64 .10$

Telex: $\quad 751194$

02 Madame Christiane Volant

07 Diplôme des Universitaire de Technologie (DUT) option Documentation d'Entreprise

! No more detailed information has been received recently.

COUNTRY

CITY, INSTITUTION:

Germany

Berlin, Freie Universität zu Berlin, Arbeitsbereich Informationswissenschaft

ACADEMIC SUBJECT: Information Science (p. 147)

01 Freie Universität Berlin

Fachbereich Kommunikationswissenschaften

Arbeitsbereich Informationswissenschaft

Malteserstr. 74-100

12249 Berlin 46

DEUTSCHLAND

Tel: $\quad+49(0) 30-77.92-8.58 / 850$

Fax: $\quad+49(0) 30-77.52-20.37$

02 Prof. Dr. Gernot Wersig (Leiter des Fachbereichs, Tel: +49(0)30-77.92-8.57)

031 Professor; 1 Oberassistent; 1 Sekretärin; 3 Stud. Hilfskräfte

04 Sommersemester 1992: Im Hauptfach: 225, im Nebenfach 142 Studierende.

05 Anwendungsprobleme wissensbasierter Systeme; Grundstrukturen der OrganisationsKommunikation; Technikfolgenabschätzung und Einsatzforschung für neue Informationsund Kommunikationstechnologien; Informationswissenschaftliche Theoriebildung; 
Bildforschung; Rationalisierung des Handelns, Lebensstile, kulturelle Entwicklungstendenzen; Museumsforschung, insbesondere Museumsmarketing; Einsatzformen von Hypermedien

06 Informationswissenschaft in Berlin 1990/91. Materialien und Hinweise zum Fach Informationswissenschaft an der Freien Universität Berlin (1991): Stand: 31.10.90. Berlin 1991

Wersig, G. (1990): Informationswissenschaft in der Bundesrepublik Deutschland - gegenwärtiger Stand und Perspektiven, in: Informatik 37, 1990, 4, 126-129

Wersig, G. (1990): Informationswissenschaft an der Freien Universität Berlin, in: Grundlagen der praktischen Information und Dokumentation. Ein Handbuch zur Einführung in die fachliche Informationsarbeit. BD. 2 München 1990, S. 1045-1053 3.Aufl. (DGD-Schriftenreihe, 9)

07 Magister Artium (MA); Doktor der Philosophie (Dr. phil) in Informationswissenschaft

! Bei der Absicht ein Studium an einer Berliner Ausbildungsinstitution zu beginnen, ist es ganz besonders wichtig aktuelle Informationen anzufordern, da zum Wintersemester 1994/95 voraussichtlich einige Institute (FU Berlin - Humboldt-Universität) fusionieren sollen!

COUNTRY:

CITY, INSTITUTION: ACADEMIC SUBJECT:

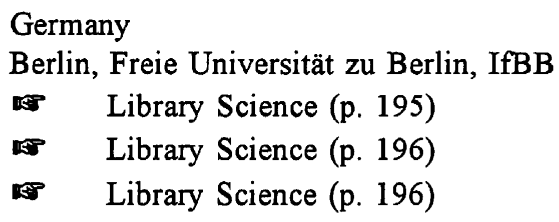

01 Freie Universität Berlin Fachbereich Kommunikationswissenschaften Institut für Bibliothekswissenschaft und Bibliothekarausbildung (IfBB) Hohenzollerndamm 56 14199 Berlin DEUTSCHLAND

Tel: $\quad+49(0) 30-8.23-69.66$

Fax: $\quad+49(0) 30-8.24-3.05 .2$

02 Prof. Dr. Peter Zahn (geschäftsführender Direktor)

037 Professoren, 27 Lehrbeauftragte

04 Wintersemester 1992/93 Diplombibliothekar/in: jeweils 93; Wintersemester 1991/92 Bibliothekswissenschaft: 362

05 Bibliothekssoziologie, Bibliotheksbetriebswirtschaftslehre, Bibliotheksinformatik, Bibliotheksgeschichte, Schrift-, Buch- und Druckgeschichte 
06 Studienordnung für die Ausbildung von Diplombibliothekaren "Dienst an wissenschaftlichen Bibliotheken" (1979), in: Mitteilungen der FU Berlin vom 17.1.1979

Studienordnung für die Ausbildung von Diplombibliothekaren "Dienst an öffentlichen Bibliotheken"(1979), in: Mitteilungen der FU Berlin vom 17.1.1979

Magisterprüfungsordnung (1982), in: Mitteilungen de FU Berlin vom 20.1.1982

Gemeinsame Promotionsordnung (1985), in: Mitteilungen de FU Berlin vom 30.12.1985

Studienhandbuch (1992): FU Berlin 1992

07 Diplombibliothekar in wissenschaftlichen Bibliotheken

Diplombibliothekar an öffentlichen Bibliotheken

Magister Artium (MA), Doktor der Philosophie (Dr. phil.) in Bibliothekswissenschaft

! Bei der Absicht ein Studium an einer Berliner Ausbildungsinstitution zu beginnen, ist es ganz besonders wichtig aktuelle Informationen anzufordern, da zum Wintersemester 1994/95 voraussichtlich einige Institute (FU Berlin - Humboldt-Universität) fusionieren sollen! Die Studiengänge der FU Berlin werden dann voraussichtlich an der HumboldtUniversität angeboten, die Immatrikulation erfolgt dort.

COUNTRY:

CITY, INSTITUTION:

Germany

Berlin, Humboldt-Universität, Institut für Bibliotheks-

wissenschaft

ACADEMIC SUBJECT: Library Science (p. 198)

01 Humboldt-Universität zu Berlin

Fachbereich Geschichtswissenschaft, Philosophie,

Bibliotheks- und Informationswissenschaft

Institut für Bibliothekswissenschaft

Clara-Zetkin-Straße 26

10117 Berlin

DEUTSCHLAND

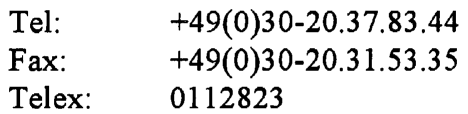

02 Prof. Dr. Schmitz (geschäftsführender Direktor) / Frau Dr. Lindner (Tel: +49(0)3020.31.52.66)

035 Hochschullehrer; 14 wissenschaftliche Mitarbeiter; 10 Fachhochschullehrer

06 Magisterstudiengang Bibliothekswissenschaft und Informationswissenschaft (1992): Humboldt-Universität zu Berlin, Institut für Bibliothekswissenschaft und wissenschaftliche Information. Berlin, Juli 1992

Verzeichnis der vereidigten Dissertationen A und B 1972-1992 (1992): HumboldtUniversität zu Berlin, Institut für Bibliothekswissenschaft und wissenschaftliche Information. Berlin, August 1992 
Chronik und bibliographisches Verzeichnis der Veröffentlichungen, Dissertationen, Diplomarbeiten und Abschlußarbeiten 1980-1990 (1991): Humboldt-Universität zu Berlin, Institut für Bibliothekswissenschaft und wissenschaftliche Information. Berlin 1991

07 Magister Artium (MA), Doktor der Philosophie (Dr. phil.) in Bibliothekswissenschaft

! Bei der Absicht ein Studium an einer Berliner Ausbildungsinstitution zu beginnen, ist es ganz besonders wichtig aktuelle Informationen anzufordern, da zum Wintersemester 1994/95 voraussichtlich einige Institute (FU Berlin - Humboldt-Universität) fusionieren sollen! Die Studiengänge der FU Berlin werden dann voraussichtlich an der HumboldtUniversität angeboten, die Immatrikulation erfolgt dort.

COUNTRY:

CITY, INSTITUTION:

ACADEMIC SUBJECT:

01 Humboldt-Universität zu Berlin Institut für Geschichtswissenschaften Lehrstuhl für Archivwissenschaft Unter den Linden 6, Postfach 1297 10117 Berlin DEUTSCHLAND

Tel: $\quad+49(0) 30-20.93-2237$

Fax: $\quad+49(0) 30-2093-27.70$

Telex: $\quad 0112823$

02 Prof. Dr. Botho Brachmann

! No more detailed information has been received recently. 


$\begin{array}{ll}\text { COUNTRY: } & \text { Germany } \\ \text { CITY, INSTITUTION: } & \text { Bielefeld, Universität Bielefeld } \\ \text { ACADEMIC SUBJECT: } & \end{array}$

01 Universität Bielefeld

Fakultät für Pädagogik

AG 9 - Informatik im Bildungs- und Sozialwesen

Universitätsstraße

33615 Bielefeld 1

DEUTSCHLAND

Tel: $\quad+49(0) 521-1.06-31.57$

Fax: $\quad+49(0) 521-1.06-58.44$

E-mail: nmeder@erasmus-nov.hrz.uni-bielefeld.de

02 Prof. Dr. Norbert Meder (+49(0)5.21-1.06-31.57)

033 Hochschullehrer; 1 wissenschaftlicher Mitarbeiter; 1 nicht-wissenschaftlicher Mitarbeiter

04 ca. 50 Studenten

05 Lernsoftware, Präsentation von Information und Wissen, Objektorientierte Lernsysteme

07 Informatiker im Bildungs- und Sozialwesen

$\begin{array}{ll}\text { COUNTRY: } & \text { Germany } \\ \text { CITY, INSTITUTION: } & \text { Bonn, Fachhochschule für das öffentliche Bibliothekswesen } \\ \text { ACADEMIC SUBJECT: } & \end{array}$

01 Postal Address: Visiting Address:

Fachhochschule für das öffentliche Bibliothekswesen Bonn (FHöBB)

Postfach $1267 \quad$ Wittelsbacherring 9

53002 Bonn 53115 Bonn

DEUTSCHLAND DEUTSCHLAND

Tel: $\quad+49(0) 228-72.58-0$

Fax: $\quad+49(0) 228-72.58-1.89$

02 Prof. Dr. Erich Hodick (Leiter); Prof. Dr. Siegfried Schmidt (Studienleiter)

031 Professor und 30 nebenamtliche Dozenten

0437 Studenten 
06 Öffentliches Bibliothekswesen. Studiengang an der Fachhochschule für das öffentliche Bibliothekswesen Bonn (1993): Fachhochschule für das öffentliche Bibliothekswesen Bonn (Hrsg.). Stand: März 1993

Diplomprüfungsordnung und Studienordnung für den Studiengang Öffentliches Bibliothekswesen (1987): Fachhochschule für das öffentliche Bibliothekswesen Bonn (Hrsg.). Stand: 30. März 1987

Schmidt, S. (1987): Gedanken zur neuen Diplomprüfungsordnung und Studienordnung der Fachhochschule für das öffentliche Bibliothekswesen Bonn für den Studiengang Öffentliches Bibliothekswesen vom 30. März 1987, in: Mitteilungsblatt 37, 1987, 3, S. 290-297

07 Diplombibliothekar für den Dienst an Öffentlichen Bibliotheken

! Die Fachhochschule für das öffentliche Bibliothekswesen Bonn blickt auf eine lange Tradition zurück. 1921 als Bonner Bibliothekarsschule gegründet, erhielt sie 1946 die staatliche Anerkennung und wurde 1984 in eine Fachhochschule umgewandelt. Träger dieser Fachhochschule ist der Borromäusverein e.V., in dessen Zentralstelle die FHöBB räumlich untergebracht ist. Die Aufgaben des Borromäusvereins liegen in der Unterstützung der katholischen Büchereiarbeit und in der Vermittlung von Büchern zu Eigenbesitz.

COUNTRY:

CITY, INSTITUTION:

Germany

ACADEMIC SUBJECT:

Darmstadt, Fachhochschule Darmstadt

Information and Documentation (p. 123)

01 Fachhochschule Darmstadt

Fachbereich Information und Dokumentation

Dekanat

Schöfferstr. 3

64295 Darmstadt

DEUTSCHLAND

Tel: $\quad+49(0) 61.51-16.84 .91$ (Sekretariat)

Fax: $\quad+49(0) 61.51-16.89 .80$ (Dekanat)

02 Prof. Dr. Klaus Löns (Dekan, Fachbereich Information und Dokumentation)

038 Professoren; 1 Labor-Ingenieur; 1 Sekretärin.

04 ca. 180 Studierende

05 Viele Kontakte; aktive Kooperation mit University of Brighton, Faculty of Information Technology, U.K. 
06 Seeger, Th. (1991): Informationsarbeit als Beruf. Erste Ergebnisse einer Befragung unter Absolventen/-innen des Fachbereichs Information und Dokumentation der Fachhochschule Darmstadt, in: Info 7, 6, 1991, 1, 26-31

07 Diplominformationswirt

$\begin{array}{ll}\text { COUNTRY: } & \text { Germany } \\ \text { CITY, INSTITUTION: } & \text { Düsseldorf, Heinrich-Heine-Universität Düsseldorf } \\ \text { ACADEMIC SUBJECT: } & \end{array}$

01 Heinrich-Heine-Universität Düsseldorf Studiengang Informationswissenschaft Universitätsstraße 1; Geb. 23.21.04

40225 Düsseldorf

DEUTSCHLAND

Tel: $\quad+49(0) 211-3.11-29.13$

Fax: $\quad+49(0) 211-3.11-34.22 .29$

E-mail: henrichs@ze8.rz.uni-duesseldorf.de / dbphilos@ze8.rz.uni-duesseldorf.de

02 Prof. Dr. Norbert Henrichs (Leiter des Studienganges, Tel: +49(0)2.11-3.11-29.13)

031 Professor; 2 Dozenten; 6 Lehrbeauftragte; 1 Programmierer; 1 Sekretärin

04 Im WS 1993/94: 345 Studenten

05 Aufbau und Pflege einer Online-Datenbank internationaler philosophischer Zeitschriftenliteratur; Automatisches Indexieren; gewichtetes Information Retrieval; Szientometrie, Hypermedia

06 Henrichs, N. (1990): Informationswissenschaft in Düsseldorf, in: Grundlagen der praktischen Information und Dokumentation. Ein Handbuch zur Einführung in die fachliche Informationsarbeit. BD. 2 München 1990, S. 1062-1072, 3. Aufl. (DGD-Schriftenreihe, 9)

07 Magister Artium (MA); Doktor der Philosophie (Dr. phil) im Nebenfach Informationswissenschaft 


$\begin{array}{ll}\text { COUNTRY: } & \text { Germany } \\ \text { CITY, INSTITUTION: } & \text { Hamburg, Fachhochschule Hamburg } \\ \text { ACADEMIC SUBJECT: } & \text { Documentation (p. 107) } \\ & \text { Library and Information Science (p. 167) }\end{array}$

01 Fachhochschule Hamburg

Fachbereich Bibliothek und Information

Grindelhof 30

20146 Hamburg 12

DEUTSCHLAND

Tel: $\quad+49(0) 40-44.19 .54 .47$

Fax: $\quad+49(0) 40-44.19 .53 .92$

02 Frau Prof. Dipl. Soz. Gudrun Bischoff-Kümmel (Fachbereichssprecherin) / Prof. Ralph Schmidt (Mediendokumentation) / Studienfachberater: Dr. Tordis Imhof-Crämer (A-K, Telefon: +49(0)40-4.44.41.95-365); Dr. Heinz Puhlmann/L-Z, Telefon: +49(0)4044.19.53.73), c) Fachschaft (Telefon $+49(0) 40-4.10 .65 .91)$

0318 Professoren; 30 nebenamtliche Lehrbeauftragte (116 SWS pro Sem.); 2 Bibliothekare; 1,5 Bibliotheksassistent; 2,5 Verwaltungsangestellte; 1 Hausmeister

04 ca. 550 Studenten

05 Benutzerforschung; Medienpädagogik; Informationsdienstleistungen

06 Hofman, U. (1993): Mediendokumentation. Ein neuer Studiengang an der Fachhochschule Hamburg, in: Bibliotheksdienst 27, 1993, 6, 893-895

Studiengang Mediendokumentation, Studienplan (1993): Fachhochschule Hamburg, FB Bibliothek und Information. Stand: April 1993

Schmidt, R. / Czudnochowski-Pelz, I. (Hrsg.)(1992): Syllabus zum Studiengang Bibliothek und Information: Kurzbeschreibungen und Erläuterungen der Studieninhalte und ziele. Hamburg: Fachhochschule Hamburg 1992.

Bischoff-Kümmel, G. (1990): Bibliothekarische Ausbildung in Hamburg 1945-1990. Hamburg

Bischoff-Kümmel, G. (1989): Muß Hamburg denn Bibliothekarinnen ausbilden?, in: Buch und Bibliothek 41, 1989, 10, 895-896

07 Diplombibliothekar; Diplomdokumentar im Studiengang Mediendokumentation 
01 Fachhochschule Hannover Fachbereich Bibliothekswesen, Information und Dokumentation Hanomagstr. 8 30449 Hannover DEUTSCHLAND

Tel: $\quad+49(0) 5.11-44.43 .44$

Fax: $\quad+49(0) 5.11-44.75 .65$

02 Prof. Dr. Jörn Klockow (Dekan)

0314 Professoren und 4 Lehrkräfte für besondere Aufgaben; 3 Verwaltungsangestellte; 4 Technische Angestellte; außerdem nehmen regelmäßig ca. 15 Lehrbeauftragte Lehraufträge wahr.

04 Ca. 450 Studenten

06 Studienführer (1993): Fachhochschule Hannover. Wintersemester 1992/93 und Sommersemester 1993

Projektarbeit im Ausbildungsbereich Bibliothekswesen, Information und Dokumentation: Erfahrungen und Anregungen (1990): Arbeitsgruppe Projektarbeit der AG-AFE. Technische Betreuung Wolfgang Hellmann [et. al.] Hannover 1990

07 Diplombibliothekar (FH); zugleich erhalten die Absolventen die Laufbahnbefähigung für den gehobenen Dienst an Wissenschaftlichen Bibliotheken.

Diplomdokumentar (FH) für Biowissenschaftliche Dokumentation

Diplomdokumentar (FH) für Allgemeine Dokumentation

Studiengang Technischer Redakteur (Diplom)

! Ausländische Bewerber wenden sich an das Institut für ausländische Fachhochschulbewerber des Landes Niedersachsen, Herrenhäuser Straße 8; D-30419 Hannover (Telefon: $+49(0) 5.11-75.44 .21)$ 


$\begin{array}{ll}\text { COUNTRY: } & \text { Germany } \\ \text { CITY, INSTITUTION: } & \text { Ilmenau, Technische Universität Ilmenau } \\ \text { ACADEMIC SUBJECT: } & \text { Information Management (p. 135) }\end{array}$

01 Technische Universität Ilmenau Institut für Wirtschaftsinformatik F6 Informationswissenschaft

Am Ehrenberg

98693 Ilmenau

DEUTSCHLAND

Tel: $\quad+49(0) 36.77-69.40 .40$

Fax: $\quad+49(0) 36.77-69.31 .52$

02 Prof. Jürgen Manecke

03 Zur Zeit noch offen.

04 Ca. 200 Studenten

05 Schwerpunkte Informationsmanagement und Informationswissenschaft

06 Manecke, J. (1991): Die Technische Hochschule Ilmenau. Eine alte (neue) Ausbildungsstätte für Informationsfachkräfte, in: Nachr. Dok. 42, 1991, 52-53

Killenberg, H. (1991): Das Konzept der informationswissenschaftlichen Ausbildung in Ilmenau, in: Wissensbasierte Informationssysteme und Informationsmanagement. Proceedings d. 2. Internationalen Symposiums f. Informationswissenschaft (ISI 91)/17. Internationales Kolloquium f. Information und Dokumenation. Konstanz 1991, 228-236 (Schriften zur Informationswissenschaft, 2), ebenso in: Informatik 38, 1991, 6, 207-208

07 Diplom Wirtschaftsinformatiker; Fachinformator

COUNTRY:

CITY, INSTITUTION:

ACADEMIC SUBJECT:
Germany

Köln, Fachhochschule für Bibliotheks- und Dokumentationswesen in Köln

Library Science (p. 201)

01 Fachhochschule für Bibliotheks- und Dokumentationswesen in Köln Claudiusstr. 1

$50678 \mathrm{Köln}$

DEUTSCHLAND

Tel: $\quad+49(0) 221-82.75-33.76$

Fax: $\quad+49(0) 221-3.31 .85 .83$ 
02 Prof. Dipl.-.Ing. Helmut Jüngling (Rektor) / Wolfgang Wesely (Studiengang ÖB, Tel: $+49(0) 2.21-82.75-33.88)$

0319 hauptamtliche oder hauptberufliche Dozenten / 27 Lehrbeauftragte

05 Forschungsprojekt Adolf von Harnack als Wissenschaftsorganisator und Bibliothekar im Rahmen seiner fachlichen Tätigkeit. Edition seiner Briefe.

06 Studienordnung für den Studiengang Öffentliches Bibliothekswesen; Diplomprüfungsordnung für den Studiengang Öffentliches Bibliothekswesen (1992): Fachhochschule für Bibliotheks- und Dokumentationswesen in Köln. Köln 1992

07 Diplombibliothekar für Öffentliches Bibliothekswesen

$\begin{array}{ll}\text { COUNTRY: } & \text { Germany } \\ \text { CITY, INSTITUTION: } & \text { Konstanz, Universität Konstanz } \\ \text { ACADEMIC SUBJECT: } & \text { Information Science (p. 150) }\end{array}$

01 Postal address:

Universität Konstanz

Visiting address:

Fachgruppe (FG) Informationswissenschaft

$\begin{array}{ll}\text { Postfach } 5560 & \text { Universitätsstr. } 10 \\ 78434 \text { Konstanz } & 78464 \text { Konstanz } \\ \text { DEUTSCHLAND } & \text { DEUTSCHLAND }\end{array}$

Tel: $\quad+49(0) 75.31-88.28 .78$

Fax: $\quad+49(0) 75.31-88.26 .01$

E-mail: kuhlen@inf.wiss.ivp.uni-konstanz.de/nagel@inf.wiss.ivp.uni-konstanz.de

02 Prof. Dr. Rainer Kuhlen (Leiter der Fachgruppe), Hans-J. Nagel (Tel: +49(0)75.3188.35.35)

033 Professoren, 1 Hochschulassistent, 6 wissenschaftliche Mitarbeiter, 1 Diplomdokumentar

04197 Studenten

05 Informationsvermittlung, Information Retrieval, Hypertext, Informationsmanagement, Wissensbasierte Systeme

06 Kuhlen, (1990): Lehre und Forschung der Informationswissenschaft an der Universität Konstanz, in: Grundlagen der praktischen Information und Dokumentation. Ein Handbuch zur Einführung in die fachliche Informationsarbeit. BD. 2 München 1990, S. 1073-1099 3. Aufl. (DGD-Schriftenreihe, 9)

Herget, J. (1989): Konzeption des Informationsmanagements in informationswissenschaftlichen Studiengängen, in: Informationsspezialisten für Europa. Die Konsequenzen des 
europäischen Binnenmarktes für die Ausbildung von Informationsfachleuten Proceedings, 17.-19. Oktober 1989. Hannover 1989, S. 165-174

Rittberger, M. (1989): Vorkenntnisse, Motivation und Arbeitsmöglichkeiten der Studenten des Aufbaustudiengangs Informationswissenschaft, in: Deutscher Dokumentartag 1989. Informationsmethoden: Neue Ansätze und Techniken 4.-6. Okt. 1989, Universität Bremen, Proceedings Frankfurt/Main 1989, S. 220-234

Kuhlen, R. (1988): Entwicklung des Faches Informationswissenschaft an der Universität Konstanz, in: R. Leibinger, H. Sund (Hrsg.) Festschrift für Lothar Späth. Universitätsverlag Konstanz 1988. S. 351-362

07 Diplominformationswissenschaftler

\author{
COUNTRY: Germany \\ CITY, INSTITUTION: Leipzig, Hochschule für Technik, Wirtschaft und Kultur \\ Leipzig (FH) \\ ACADEMIC SUBJECT: Library Science (p. 202)
}

01 Hochschule für Technik, Wirtschaft und Kultur Leipzig (FH)

Fachbereich Buch und Museum

Berthold-Brecht-Straße 1

04347 Leipzig

DEUTSCHLAND

Tel: $\quad+49(0) 3.41-2.31 .12 .02$

Fax: $\quad+49(0) 3.41-2.31 .12 .80$

Telex: $\quad 311222$

02 Prof. Dr. jur. Engelbert Plassmann (Dekan, Tel. +49(0)3.41-2.31.12.02); Studienfachberatung Prof. Dietmar Kummer (Tel: $+49(0) 3.41-2.31 .12 .02)$

039 Professoren im Studiengang Bibliothekswesen, 6 wissenschaftliche Mitarbeiter (davon 2 im Studiengang Bibliothekswesen), 5 nichtwissenschaftliche Mitarbeiter (davon zwei als Halbtagskräfte), das Lehrdeputat für Professoren beträgt 18 SWS.

04 Im Studiengang Bibliothekswesen (am 1.3.1993): 464 (davon 274 Fernstudenten in auslaufenden Studiengängen; ein Fernstudium ist nicht mehr möglich)

05 Auf Grund der noch relativ kurzen Existenz des Fachbereichs Buch und Museum haben sich derzeit noch keine wirklichen Forschungsschwerpunkte herausgebildet. Akzente der Forschung liegen auf folgenden Gebieten: Bibliotheksmarketing, Benutzer- und Benutzungsforschung, Arbeit in Kinder- und Jugendbibliotheken, Deutsche Bibliotheksgeschichte des 20. Jahrhunderts

06 Plassmann, E. / Seela, T. (1993): Bibliothekarisches Studium in Leipzig. ZfBB 40, 1993, $1,86-88$ 


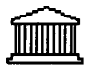
$\underline{42}$

Schlegel, J. (1993): DV-Ausbildung im Fachbereich Buch und Museum der neugegründeten Hochschule für Technik, Wirtschaft und Kultur Leipzig (FH). Bibliotheksdienst 27, 1993, 5, 695-702.

07 Diplombibliothekar

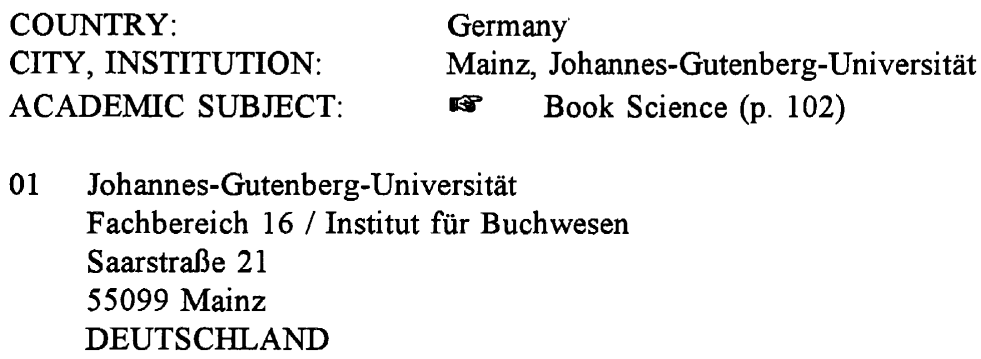

01 Johannes-Gutenberg-Universität

Fachbereich 16 / Institut für Buchwesen

Saarstraße 21

55099 Mainz

DEUTSCHLAND

Tel. $\quad+49(0) 6131-39.25 .80$

Fax: $\quad+49(0) 6131-39.34 .22$

02 Prof. Dr. Stephan Füssel

032 Professoren; 4 wissenschaftliche Mitarbeiter und 3 nicht-wissenschaftliche Mitarbeiter

04718 Studenten, davon 270 Hauptfach-Studenten

05 Verlagsgeschichte um 1800; Buchhandelsgeschichte in der Weimarer Republik; Inkunabelforschung

07 Magister Artium (MA), Doktor der Philosophie in Buchwissenschaft

! Dies ist der einzige Lehrstuhl für Buchwissenschaft im deutschprachigen Raum.

COUNTRY:

CITY, INSTITUTION: $\quad$ Potsdam, Fachhochschule Potsdam

ACADEMIC SUBJECT: Information and Documentation (p. 110)

01 Fachhochschule Potsdam

Fachbereich ABD

Friedrich-Ebert-Str. 4

14467 Potsdam

DEUTSCHLAND

Tel: $\quad+49(0) 331-2.88 .42 .14$ 
02 Prof. Schuler (Fachbereichsleiter)

07 Diplomdokumentar (FH)

COUNTRY:

CITY, INSTITUTION:

ACADEMIC SUBJECT:

\author{
Germany \\ Potsdam, Institut für Information und Dokumentation (IID) \\ $\infty$ Information and Documentation (p. 125)
}

01 Postal address

Visiting address

Institut für Information und Dokumentation (IID)

Postfach 601553

Am Neuen Palais 10, Gebäude I, R. 129

14415 Potsdam

14469 Potsdam

DEUTSCHLAND

DEUTSCHLAND

Tel: $\quad+49(0) 331-9.77 .11 .02$

Fax: $\quad+49(0) 331-97.21 .63$

02 Prof. Dr. Harald Milloning (Leiter des Instituts)

031 Professor, 4 Dozenten, 1 Labor-Leiter, 1 Sachbearbeiter, 1 Sekretär

04 Ca. 50 Studenten pro Jahr

05 Ausbildungs- und Berufsfeldforschung im Informationswesen

06 Informationen zur berufsbegleitenden Ausbildung zum/zur wissenschaftlichen Dokumentar/in (1992): Fachhochschule Potsdam, Institut für Information und Dokumentation (IID). Potsdam, Oktober 1992

Einführungsmaterialien zur berufsbegleitenden Ausbildung zum/zur wissenschaftlichen Dokumentar/in (1992): Lehrinstitut für Information und Dokumentation Potsdam. Potsdam, März 1992

Seeger, Th. (1993): Erste wissenschaftliche Dokumentare und Dokumentarinnen aus Potsdam, Nach. Dok. 44, 1993, 121

Strauch, D. (1993): Wissenschaftlicher Dokumentar und wissenschaftliche Dokumentarinnen staatlich anerkannt, Nach. Dok. 44, 1993, 120-121.

07 Wissenschaftlicher Dokumentar 
COUNTRY

CITY, INSTITUTION:

ACADEMIC SUBJECT:

\author{
Germany \\ Regensburg, Universität Regensburg \\ Information Science (p. 152)
}

01 Postal adress

Visiting address:

Universität Regensburg

Linguistische Informationswissenschaft

Postfach 101042

Universitätsstr. 32

93040 Regensburg 93053 Regensburg

DEUTSCHLAND DEUTSCHLAND

Tel: $\quad+49(0) 941-9.43-41.95$

E-mail: hitz@vax1.rz.uni-regensburg.dpe.de

02 Dr. L. Hitzenberger

04 ca. 100 Studenten

05 Mensch-Maschine-Kommunikation, Software-Ergonomie, Information Retrieval, gesprochene Sprache, Evaluierung

06 Hitzenberger, L. / Krause, J. / Schneider, C. (1986). Die linguistische Informationswissenschaft an der Universität Regensburg, Sprache und Datenverarbeitung; International Journal for Language Data Processing 10, 1986, 2, 52-55

07 Magister Artium (MA); Promotion in linguistischer Informationswissenschaft

COUNTRY:

CITY, INSTITUTION:

ACADEMIC SUBJECT

\author{
Germany \\ Saarbrücken, Universität des Saarlandes \\ Information Science (p. 152)
}

01 Universität des Saarlandes

FR. 5.5 Informationswissenschaft

Gebäude 4

66041 Saarbrücken

DEUTSCHLAND

Tel: $\quad+49(0) 681-3.02 .35 .37$

Fax: $\quad+49(0) 681-3.02 .35 .57$

E-mail: infowiss@rz.uni-sb.de / zimmermann@rz.uni.sb.de / gg15hzfi@rz.uni-sb.de (Fachschaft)

02 Prof. Dr. Harald H. Zimmermann (Leiter der Fachrichtung); Valentin von Dietmann (Zentrale Studienberatung der Universität des Saarlandes, Tel +49(0)6.81-3.02-36.83, Email: zsbusvd@rz.uni-sb.de) 
031 Professor/1,5 wissenschaftliche Mitarbeiter (Dr. Ilse Harms, Angelika Schulz, M.A./ div. Lehraufträge/0,5 nicht-wissenschaftliche Mitarbeiter (19 Wochenstunden Bibliothek).

04 Im Studienjahr WS 92/93: 524 Studierende (Haupt- und Nebenfach)

05 Forschungsschwerpunkte: Beteiligung am WINGS-Projekt. WINGS-Wissensvermittlung und Informationstechnologien in den Geistes- und Sozialwissenschaften. Ziel des Projekts ist es, zu einer fachintegrierten Nutzung der Informationstechnologien im Bereich der Philosophischen Fakultät zu qualifizieren (in Kooperation mit der Universität Potsdam). Computervermittelte Kommunikation in der Wissenschaft: Einsatzmöglichkeiten, Nutzungsmodalitäten, Akzeptanz und Auswirkungen im Rahmen der wissenschaftlichen Kommunikation.

06 Magisterprüfungsordnung (1985): Ordnung für die Akademische Abschlußprüfung (Magisterprüfung) der Philosophischen Fakultät der Universität des Saarlandes (Magisterprüfungsordnung) vom 14. Dezember 1985 (Dienstbl. S. 162) mit Änderung vom 23. April 1985 (Dienstbl. S. 168) und Berichtigung vom 14. April 1986 (Dienstbl. S. 164) Promotionsordnung (1985: der Philosophischen Fakultät der Universität des Saarlandes vom 15. Mai 1985 (Dienstbl. S. 150) mit Änderung vom 14. Januar 1987 (Dienstbl. S. 30)

Studienplan Informationswissenschaft (4.10.84)

Studienordnung (1984): des Studienganges Informationswissenschaft im Rahmen des Magisterstudiums der Philosophischen Fakultät vom 11. Juli 1984 (Dienstbl. S. 138)

Harms, I. / Schulz, A. (1991): Informationswissenschaft in Saarbrücken - Stand und Perspektiven, in: Wissensbasierte Informationssysteme und Informationsmanagement. Proceedings d. 2. Internationalen Symposiums f. Informationswissenschaft (ISI 91)/17. Internationales Kolloquium f. Information und Dokumentation. Konstanz 1991, 237-246 (Schriften zur Informationswissenschaft, 2)

Zimmermann, H. (1990): Informationswissenschaft an der Universität des Saarlandes ("Saarbrücker Modell"), in: Grundlagen der praktischen Information und Dokumentation. Ein Handbuch zur Einführung in die fachliche Informationsarbeit. B. 2. München 1990, 1100-1107 3. völlig neu gef. Ausg. (DGD-Schriftenreihe, 9)

Zimmermann, H. (1988): Studienführer zum Fach Informationswissenschaft. Universität des Saarlandes, Fachrichtung 5.5 Informationswissenschaft, 1988 (Arbeitspapier für Studierende)

Weitere Informationen: siehe auch im elektronischen Informationssystem Gopher unter Fachrichtung Informationswissenschaft (Internet: pfsparc02.phil15.uni-sb.de, Telnet: 134.96.82.13)

07 Magister Artium (MA); Doktor der Philosophie (Dr. phil., Promotion als 1. Studienabschluss und nach abgeschlossenem Hochschulstudium möglich) in Informationswissenschaft 
COUNTRY:

CITY, INSTITUTION:

ACADEMIC SUBJECT:

\section{Germany}

Stuttgart, Fachhochschule für Bibliothekswesen

Documentation (p. 111)

Library Science (p. 203)

01 Fachhochschule für Bibliothekswesen

Feuerbacher Heide 38-42

70192 Stuttgart

DEUTSCHLAND

Tel: $\quad+49(0) 711-22.74 .20$

Fax: $\quad+49(0) 711-2.27 .42 .33$

02 Prof. Dr. Peter Vodosek (Rektor), Prof. Dr. Askam Blum (Prorektor)

0329 Professoren, 3 Hochschulassistenten, 1 Diplombibliothekar, 1 techn. Leiter des Rechenzentrums (Dipl.-Bib.), 2 Verwaltungsbeamte, 10 Verwaltungsangestellte

04 Insgesamt 442, öffentliche Bibliotheken: 360, Dokumentation: 72

06 Studienführer (1991): Fachhochschule für Bibliothekswesen Stuttgart. 5. Aufl., Stuttgart 1991

Das Studium an der FHB Stuttgart. Informationsbroschüre (1991): Stuttgart 1991

07 Diplombibliothekar; Diplomdokumentar; Musikbibliothekar an öffentlichen Bibliotheken

COUNTRY:

CITY, INSTITUTION: Budapest, Eötvös Loránd Tudományegyetem (Lorand Eötros

ACADEMIC SUBJECT: University Budapest)

01 Eötvös Loránd Tudományegyetem

(Lorand Eötros University Budapest)

Philogical Faculty

Department of Library and Information Studies

Múzeum Körut 6-8

1088 Budapest

MAGYARORSZÁG

Tel: $\quad+36(0) 2-66.79 .46$

Fax: $\quad+36(0) 2-26.79 .46$

Telex: 225467

02 Dr. Géza Fülop (Head of Department) 
03 Full-time staff: 7 teachers, 1 librarian, 1 secretary; part-time: 3 teachers, 1 technician; external lecturers: 20 persons

$04 \quad 100-110$ students

05 History of the Books and Libraries in Hungary; Library Administration and Management; Information, Bibliography and Documentation

07 Bachelor of Arts (BA), Master of Arts (MA), Doctor of Philosophy (PhD) in Library and Information Studies

! Beside the day-course we have a second-diploma course and postgraduate courses too.

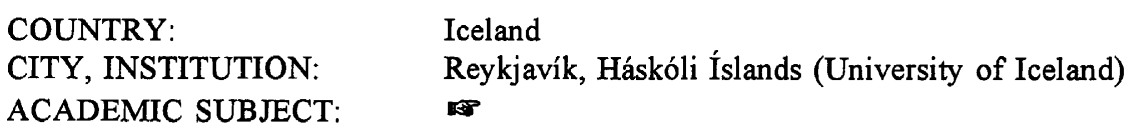

01

(University of Iceland)

Department of Library and Information Studies

Sudurgata

101 Reykjavík

ÍSLANDS

Tel: $\quad+354(0) 1-69.43 .00$

Fax: $\quad+354(0) 1-2.13 .31$

Telex: $\quad 2307$

! No more detailed information has been received recently.

$\begin{array}{ll}\text { COUNTRY: } & \text { Ireland } \\ \text { CITY, INSTITUTION: } & \text { Dublin, University College Dublin (UCD) } \\ \text { ACADEMIC SUBJECT: } & \text { Information Studies (p. 160) }\end{array}$

01 University College Dublin (UCD)

Department of Library and Information Studies

Belfield

Dublin 4

IRELAND

Tel: $\quad+353(0) 1-7.06 .70 .80$

Fax: $\quad+353(0) 1-7.06 .70 .55 / 69.44 .09$

Telex: $32693 \mathrm{EI}$ 
02 Dr. Mary Burke (Phone: $+353(0) 1-7.06 .70 .80$ )

036 full-time academic staff; 10 part-time lecturers and tutors

0425 Students p.a.

05 Information Retrieval; Foundations of Information Science; Information Market; Multimedia; Historical Bibliography

07 Master of Arts (MA) in Information Studies

! The following outline of living expenses is intended to give prospective students an indication of the cost of living in Ireland. It should be emphasized that the data quoted are approximate and serve as a guide only (costs per Academic Year $=9$ Months) Accomodation $=$ IR $£ 1.350$-IR $£ 1.800 ;$ Food $=$ IR $£ 1.500$; Entertainment and other living expenses $=\mathrm{IR} £$ 800-IR $£$ 1.000; Textbooks and Study Materials $=$ IR $£ 200$

COUNTRY: Italy

CITY, INSTITUTION: $\quad$ Ravenna, Università degli Studi di Bologna, sede di Ravenna ACADEMIC SUBJECT: Archive Science (p. 96)

01 Postal address

Università degli Studi di Bologna

Visiting address

Scuola diretta a fini speciali per archivisti

Via Zamboni 33

Palazzo del Seminario

40126 Bologna

Piazza Duomo

ITALIA

Tel: $\quad+39(0) 5.44-35.46 .81 / 82.83 .84$

Fax: $\quad+39(0) 5.44-22.87 .21$

02 Prof. Antonio Carile (Head of Department)

03 Teaching staff: 12 Professors

06 Vespignani, G. (1991): Scuola diretta a fini speciali per Archivisti. Università degli Studi di Bologna, Sede di Ravenna 1991

07 Diploma in Archivistica 


$\begin{array}{ll}\text { COUNTRY: } & \text { Italy } \\ \text { CITY, INSTITUTION: } & \text { Roma, Università degli Studi di Roma "La Sapienza" } \\ \text { ACADEMIC SUBJECT: } & \end{array}$

01 Università degli Studi di Roma "La Sapienza"

Scuola speciale per Archivisti e Bibliotecari

Via Vicenza 23

00185 Roma

ITALIA

Tel: $\quad+39(0) 6-4.45 .43 .35 / 4455377$ (Centralino)

Tel: $\quad+39(0) 6-4.45 .67 .96$ (Presidenza, Segreteria)

Fax: $\quad+39(0) 6-4.45 .67 .96$ (Presidenza, Segreteria)

02 Prof. Dr. Elio Lodolini (Preside della Scuola)

03 Academic staff: 20

04 Circa 150 studenti

06 Ordine degli Studi. Anno Accademico 1992-1993 (1992): Università degli Studi di Roma "La Sapienza". Scuola speciale per Archivisti e Bibliotecari. Roma 1992

07 Diploma per Bibliotecaria

! La Scuola speciale per Archivisti e Bibliotecari è sede di promozione e di sviluppo della ricerca scientifica nell'ambito delle discipline archivistiche, biblioteconomiche e documentarie.

COUNTRY:

CITY, INSTITUTION:

ACADEMIC SUBJECT:

\author{
Latvia \\ Riga, Latvijas Universitate \\ Library and Information Studies (p. 175)
}

01 Postal address:

Visiting address:

Latvijas Universitate (University of Latvia)

Department of Library and Information Studies
4 A Visvalzha St.
Raina boulevard 19
LV 1011 Riga
Riga
LATVIJA
LATVIJA

Tel: $\quad+7(0) 1-32.22 .50 .13$

Fax: $\quad+7(0) 1-32.22 .50 .39$

02 Solveiga Skotele (Head of Library and Information Studies Department, Tel. $+7(0) 1$ 22.44.53) 
03 Full-time staff-members: 4 doctors in education, 4 lecturers in library and information studies. part-time staff-members: 2 doctors and 3 lecturers

04260 students

05 Thesaurus construction in Library Science in Latvia.

07 Bachelor of Arts (BA), Master of Arts (MA) in Library and Information Studies Diploma in Librarianship

$\begin{array}{ll}\text { COUNTRY: } & \text { Lithuania } \\ \text { CITY, INSTITUTION: } & \text { Vilnius, U }\end{array}$

ACADEMIC SUBJECT: Library and Information Science (p. 176)

01 University of Vilnius

Faculty of Communication

Saulétekio 9

2054 Vilnius

LIETUVA

Tel: $\quad+(7) 01.22-76.89 .53$

Fax: $\quad+(7) 01.22-61.34 .73$

02 Renaldas Gudauskas (Dean of Faculty)

0345 full-time, 15 part-time

04500 students, 100 p.a.

05 Library and Information Science, Book Science, Theory of Communication

06 Vilnius University Information (1992): Vilnius University Press 1992

07 Bachelor of Arts (BA), Master of Arts (MA) in Library and Information Science 


$\begin{array}{ll}\text { COUNTRY: } & \text { The Netherlands } \\ \text { CITY, INSTITUTION: } & \text { Amsterdam, Hogeschool van Amsterdam } \\ \text { ACADEMIC SUBJECT: } & \quad \text { Library and Information Science (p. 176) }\end{array}$

01 Postal address

Hogeschool van Amsterdam

Visiting address

Faculteit Economie en Informatie

Postbus 10895

Opleiding Bibliotheek en documentaire Informatie (BDI)

1001 EW Amsterdam

NEDERLAND

Herengracht 330

1016 CE Amsterdam

NEDERLAND

Tel: $\quad+31(0) 20-6.26 .51 .55$

Fax: $\quad+31(0) 20-6.23 .88 .69$

02 Prof. Ruud Bruyns (Head of Department) / Gerard J. Wijnands / Barry Wiebenga (Tel: $+31(0) 20-6.26 .51 .55)$

0316 full-time and 13 part-time scientific employees

04300 students

05 Library and Information Science

07 Bachelor in Library and Information Science

! Partner for address for foreign students: Mrs. K. van der Wissel, International Services, Amsterdam Polytechnic, Europaboulevard 23, NL-1079 PC Amsterdam (Tel: +31(0)206.42.93.33) 
血监 52

COUNTRY:

The Netherlands

CITY, INSTITUTION:

Amsterdam, Universiteit van Amsterdam

ACADEMIC SUBJECT

Information Science (p. 154)

01 Universiteit van Amsterdam

Department of Book, Library and Information Science

Singel 425

1012 WP Amsterdam

NEDERLAND

Tel: $\quad+31(0) 20-5.25-20.51$

Fax: $\quad+31(0) 20-5.25-23.11$

E-mail: albertkb@sara.nl

02 Albert K. Boekhorst

032,7 scientific staff; 0,4 administrative staff

0450 students

05 Information Literacy; Information Use, Information Languages

07 Masters Degree, Certificates in Book and Information Science 


$\begin{array}{ll}\text { COUNTRY: } & \text { The Netherlands } \\ \text { CITY, INSTITUTION: } & \text { Deventer, Rijkshogeschool IJselland } \\ \text { ACADEMIC SUBJECT: } & \text { Business Information (p. 102) } \\ & \text { Library Science (p. 205) }\end{array}$

01 Postal address:

Visiting address:

Rijkshogeschool IJselland

Bibliotheek en Documentaire Informatieverzorging (BDI)

Postbus 333

7400 AH Deventer

NEDERLAND

7423 EL Deventer

NEDERLAND

Tel: $\quad+31(0) 57.00-5.20 .00$

Fax: $\quad+31(0) 57.00-5.19 .39$

E-mail: riek.oxener@heo.rhij.nl

02 Mevr. Riek A. Oexener

03 Academic staff: 16 (full-time and part-time)

04 Library Science: 160; Business Information: 83

06 Study Guide 1993-1994 (1993): Programme in Library Studies (LS). Full-time course. State Polytechnic IJselland, Faculty of Higher Economic Education, Department of Library and Information Studies (LS). 1993

Study Guide 1993-1994 (1993): Programme in Business Information. Full-time course. State Polytechnic IJselland, Faculty of Higher Economic Education, Department of Library and Information Studies (LS). 1993

07 Diploma in Library Science / Documentation

Diploma in Business Information 


\section{COUNTRY: $\quad$ The Netherlands \\ CITY, INSTITUTION: $\quad$ Groningen, Rijkshogeschool Groningen \\ ACADEMIC SUBJECT:}

01 Rijkshogeschool Groningen

Bibliotheek, Documentatie en Informatieverzorging

Veldspaatsstraat 1

9704 CM Groningen

NEDERLAND

02 A. M. Ferrari

! No more detailed information has been received recently.

COUNTRY:

CITY, INSTITUTION:

The Netherlands

ACADEMIC SUBJECT:

Maastricht, Rijkshogeschool Maastricht

Information Resource Management (p. 136)

Library and Information Science (p. 177)

01 Postal address

Rijkshogeschool Maastricht

Faculteit Economische Dienstverlening

Postbox 634

Brusselseweg 150

6200 AP Maastricht

$6217 \mathrm{HB}$ Maastrich

NEDERLAND NEDERLAND

Tel: $\quad+31(0) 43-46.66 .10$

Fax: $\quad+31(0) 43-46.66 .19$

02 Hans Koolmees (Dean, Tel: $+31(0) 43-46.66 .12)$

0311 full-time and 9 part-time staff (lecturers); 2 administrative staff; 2 persons for computer support; 6 staff in mediathek and 5 others

04250 students

05 Hypermedia

07 Hoger Beroepsonderwijs (HBO) in Library and Information Science Hoger Beroepsonderwijs (HBO) in Information Resource Management 


\section{COUNTRY: $\quad$ The Netherlands \\ CITY, INSTITUTION: Tilburg, Hogeschool Midden-Brabant \\ ACADEMIC SUBJECT:}

01 Hogeschool Midden-Brabant

Prof. Cobbenhagenlaan 13

Postbus 1097

5004 BB Tilburg

NEDERLAND

Tel: $\quad+31(0) 13-63.52 .50$

Fax: $\quad+31(0) 13-63.79 .42$

02 Mrs. Dolly van Rossem

07 Library and Information Science

! No more detailed information has been received recently.

$\begin{array}{ll}\text { COUNTRY: } & \text { Northern Ireland } \\ \text { CITY, INSTITUTION } & \text { Belfast, Queen's University of Belfast } \\ \text { ACADEMIC SUBJECT: } & \end{array}$

01 Queen's University of Belfast

School of Finance and Information

Information Management Division

Belfast BT7 1NN

NORTHERN IRELAND

Tel: $\quad+44(0) 232-24.51 .33-50.11$

Fax: $\quad+44(0) 232-32.86 .49$

Telex: $\quad 74487$

E-mail: j.warner@v2.qub.ac.uk

02 Prof. K. D. Brown (Director) / Dr. Julian Warner

031 professor, 3 senior lecturers, 7 lecturers, 2 secretarial staff, 1 computer network manager

0445 students

05 Information Management, Total Quality Management, Semiotics, Information Retrieval 
06 Diploma in Information Management (1993): The Queen's University of Belfast, School of Finance and Information, Information Management Division. 1. February 1993.

07 Master of Science (MSc), Diploma in Information Management

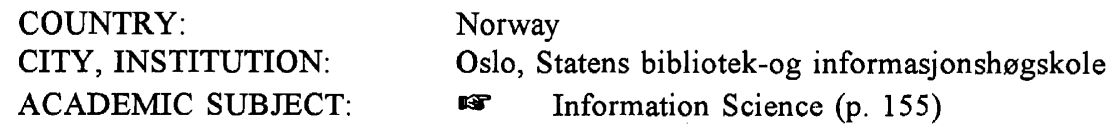

01 Statens bibliotek-og informasjonshøgskole

Norwegian School of Library and Information Science

Dælenenggt. 26

0567 Oslo

NORGE

Tel: $\quad+47(0) 2-35.73 .90 /-35.02 .66$

Fax: $\quad+47(0) 2-35.12 .60$

02 Tor Henriksen (Rektor)

03 staff of 50 persons; $2 / 3$ of them of a full time basis

04420 full-time students equivalents

05 We do research and development projects in common, exchange information on curricula and discuss them.

06 Henriksen, T. (1990): Education, Research and Profession, in: Library Automation and Networking: European Conferences. 9-11 May 1990. Brussels 1990

Alnaes, T. (1988): Library and Information Science Education in Norway, in: Education for Information, 1988, 6, 187-197

Henriksen, T. (1989): Bibliotek- og Informasjonsutdanning i Norge, in: Nordinfo Nytt, $12,1989,32-38$

07 Information Science and Data Processing (postgraduate)

! In 1994, the school will be integrated with about 20 others to form a Polytechnic for the Oslo and Akershus region. 


$\begin{array}{ll}\text { COUNTRY: } & \text { Poland } \\ \text { CITY, INSTITUTION: } & \text { Gdańsk, Uniwersytet Gdański } \\ \text { ACADEMIC SUBJECT: } & \end{array}$

01 Uniwersytet Gdański

Book Research Department

Library Management

Ul. Wita Stwosza 55

80-952 Gdańsk

POLSKA

Fax: $\quad+48-(0) 58-52.03 .11$

02 Prof. dr. Wiktor Peplinski

! No more detailed information has been received recently.

COUNTRY:

Poland

CITY, INSTITUTION:

Kraków, Uniwersytet Jagiellónski

ACADEMIC SUBJECT:

Information Science (p. 155)

Library and Information Science (p. 178)

Library Science (p. 206)

01 Uniwersytet Jagiellónski (The Jagiellonian University)

Katedra Bibliotekoznawstwa i Informacji Naukowej

(Department of Librarianship and Information Science)

ul. Gołębia 16

31-0076 Kraków

POLSKA

Tel: $\quad \quad+48(0) 12-22.10 .33-4.20 / 3.23$

Fax: $\quad+48(0) 12-22.63 .06-22.17 .57$

02 Prof. dr. hab. Stanisław Grzeszczuk (Head of Department)

032 full-professors; 3 associate professors; 6 doctors; 4 assistants, 1 computer officer; 2 part-time teaching staff; 1 technical staff; 1 administrative staff; ca. 30 guest lecturers

04 1991: 223 students

05 The research work of the Department of Librarianship and Information Science is concentrated mainly in the border regions of Librarianship, Information Science, Documentation, Bibliology and Social Communication, Sociology, Psychology, Economy, Culture, etc. The library as social institution provides a reference point for our research 
work. The research tends to follow three basic directions: a) Books, Libraries and Information Science in the process of social communication, b) problems of theory, methodology and education of Bibliology, Librarianship and Information Science, c) source and methodological research on Bibliology, Librarianship and Information Science.

06 The Department of Librarianship and Information Science (1992): The Jagiellonian University. Cracow, Poland. Crakow 1992

07 Master in Library and Information Science

Diploma in Library Science

Diploma in Information Science

! This department is one of the important departments of Librarianship in Poland. The study of this discipline has a long tradition at the Jagiellonian University. Lectures in Bibliography were inaugurated here in 1811. - Foreign students are expected to meet the same requirements as Polish students, which means that they have to be both spoken and written Polish. Students from abroad have to pay annual fees which are fixed by the Jagiellonian University.

$\begin{array}{ll}\text { COUNTRY: } & \text { Poland } \\ \text { CITY, INSTITUTION: } & \text { Kraków, Wyższa Szkoła Pedagogiczna im. KEN } \\ \text { ACADEMIC SUBJECT: } & \end{array}$

01 Wyższa Szkoła Pedagogiczna im. KEN (Komisji Edukacji Narodowej) w Krakowie Katedra Bibliotekoznawstwa i Informacji Naukowej

Ulica Ingardena 4

30-060 Kraków

POLSKA

Tel: $\quad+48(0) 12-33.84 .25$

02 Prof. Dr hab. Jerzy Jarowiecki (tel. domowy +48(0)12-11.08.74)

03 Pracowniy naukowi 24 i nienakowi 5.

04 Studenci stacjonarni 229 i zaoczni 111. 


$\begin{array}{ll}\text { COUNTRY: } & \text { Poland } \\ \text { CITY, INSTITUTION: } & \text { Poznań, Uniwersytet im Adama Mickiewicza w Poznaniu } \\ \text { ACADEMIC SUBJECT: } & \end{array}$

01 Uniwersytet im Adama Mickiewicza w Poznaniu

(Adam Mickiewicz University in Poznań)

Institute of Library and Information Science

Ul. Heuryka Wieniawskiego

61 - 712 Poznań

POLSKA

Tel: $\quad+48(0) 61-6.99-2.51 / 52.64 .25$

Fax: $\quad+48(0) 61-5.35-5.35$

Telex: $\quad 0413260$

! No more detailed information has been received recently.

$\begin{array}{ll}\text { COUNTRY: } & \text { Poland } \\ \text { CITY, INSTITUTION: } & \text { Torun, Uniwersytet Mikolaja Kopernika } \\ \text { ACADEMIC SUBJECT: } & \end{array}$

01 Uniwersytet Mikolaja Kopernika

Wydzial Humanistyczny

Instytut Histori i archiwistyki

Zakład bibliotekoznawstwa i Informacji Naukowej

(Department of Library and Information Science)

Ul. Gagarina 13

87-100 Toruń

POLSKA

Tel: $\quad+48(0) 56-1.44 .14 / 1.44 .80$

Fax: $\quad+48(0) 56-48.56 / 2.33 .52$

Email : umklibr@pltumk.pl

02 Prof. dr. hab. Bohdan Ryszewski (Tel: +48(0)56-1.44.15)

03 Pracownicy naukowi (pełnoetatowo zatrudnieni), 9; Pracownicy naukowi (półetatowo zatrudnieni), 4; Nienaukowi pracownicy (pełnoetatowo zatrudnieni), 1

04 Ogólna liczba studentów-252 osoby (studia stacjonarne: 169, studia zaoczne: 83 )

05 Historia książki i bibliotekarstwa; Aktywność publicystyczna i czytelnictwo na Pomorzu w XIX wieku; Systemy informacyjne w bibliotekach naukowych; Skomputeryzowane bibliografie historyczne. 
07 Master in Library and Information Science

! Zaliczenia większości przedmiotów na podstawie rocznych a nie semestralnych kursów.

COUNTRY:

CITY, INSTITUTION:

ACADEMIC SUBJECT:
Poland

Warszawa, Uniwersytet Warszawski

Library and Information Science (p. 181)

01 Uniwersytet Warszawski

Wydzial Historyczny

Instytut Bibliotekoznawstwa i Informacji Naukowej UW

(Department of Library and Information Science)

ul. Nowy Świat 69

00-046 Warszawa

POLSKA

Tel: $\quad+48(0) 22-26.85 .69$

Fax: $\quad+48(0) 22-26.85 .69$

E-mail: ibin@plearn.bitnet / instbibl@frodo.nask.org.pl

02 Prof. Dr. hab. Marcin Drzewiecki (Direktor); Dr. Barbara Sosińska-Kalata; Wieslaw Glinski

0330 Dozenten; 6 technische Mitarbeiter

04500 Studenten

05 Die Abteilung Informationssysteme hat folgende Forschungsschwerpunkte: Information Retrieval innerhalb von Bibliotheksanwendungen; Datenbanken; Wissensbasierte Systeme; Terminologische Datenbanken; Benutzerschnittstellen; Expertensysteme; Computerlinguistik; Künstliche Intelligenz; Wissensrepräsentation.

06 Zybert, E. B. (1991): Warsaw University. Institute of Librarianship and Information Science. Organization, Research and Educational Programs (translated by Stefan Skora). Instytut Bibliotekoznawstwa i Informacji Naukowej UW 1991

07 MA (full-time and extramural); Diploma in Information Science for Teachers (postgraduate)

$1 \quad$ Ein Informations- und Dokumentationszentrum über Kinderliteratur sowie ein Beratungszentrum für die Automatisierung von Bibliotheken soll aufgebaut werden. 


$\begin{array}{ll}\text { COUNTRY: } & \text { Poland } \\ \text { CITY, INSTITUTION: } & \text { Wrocław, Politechnika Wrocławska } \\ \text { ACADEMIC SUBJECT: } & \end{array}$

01 Politechnika Wrocławska

Department of Information Systems

Wybrzeze St. Wyspiańskiego 27

50-379 Wrocław

POLSKA

Tel: $\quad+48(0) 71-20.23 .58$

Fax: $\quad+48(0) 71-21.19 .60$

E-mail: depis@plwrtu.pl

02 Doc. Dr. inż C. Daniłowicz; Joanna Cieślik (Tel: +48(0)71-20.37.99)

03 Scientific employees: 17; Non-scientific employees: 3

04150 students

05 New methods of documents content representation in information retrieval systems; methods of representation of scientific problems in the information retrieval systems; methods of compressing bibliographic data; methods of choice of partitions and covering representation; recognizing hypertext methods and designing hypertext-relational information retrieval systems

07 Master of Science in Information Systems

$\begin{array}{ll}\text { COUNTRY: } & \text { Poland } \\ \text { CITY, INSTITUTION: } & \text { Wrocław, Uniwersytet Wrocławski } \\ \text { ACADEMIC SUBJECT: } & \end{array}$

01 Uniwersytet Wrocławski

Wydzial Filologiczny

Instytut Bibliotekoznawstwa

Pl. Uniwersytecki 9-13

50-137 Wrocław

POLSKA

Tel: $\quad \quad+48(0) 71-3.78 .11 / 40.24 .12$

Fax: $\quad+48(0) 71-40.28 .62$

E-Mail: arp@plwruw11.earn

02 Renata Aleksandrowicz (Tel: $+48(0) 71-40.28 .61)$ / Aleksander Radwanski 
03 Scientific full-time staff: 28 ; scientific part-time staff: 3 ; non-scientific staff: 6

04 full-time students 200; part-time students 120

05 Theory and Methodology of Book Science, Printing History, Reading and Book Art, Information Science

07 Master of Arts (MA) in Library and Information Science Study

$\begin{array}{ll}\text { COUNTRY: } & \text { Portugal } \\ \text { CITY, INSTITUTION: } & \text { Coímbra, Universidade de Coímbra } \\ \text { ACADEMIC SUBJECT: } & \end{array}$

01 Universidade de Coímbra

Faculdade de Letras

Largo de D. Dinis

3049 Coímbra Cedex

PORTUGAL

Tel: $\quad+351(0) 39-2.61 .48 / 2.51 .10$

Telex: $\quad 52273$

02 Prof. Doutor Anibal Pinto de Castro

033 enseignants à plein temps et 8 à temps partiel.

0434 étudiants

06 Reference of this item: International Council on Archives (1992): Répertoire des Ecoles et des cours de formation professionelle d'Archivistes/Directory of schools and courses of professional training for archivists. (Studies études, 6). Conseil International des Archives / International council on Archives. Koblenz 1992.

07 Diplôme du cours specializaçào em ciencias documentais 
COUNTRY:

CITY, INSTITUTION:

ACADEMIC SUBJECT:
Portugal

Lisbon, Instituto Nacional de Engenharia e Tecnologia Industrial (INETI)

Information Management (p. 138)

01 Instituto Nacional de Engenharia e Tecnologia Industrial (INETI)

Centro de Informação Técnica para a Indústria (CITI)

Azinhaga dos Lameiros à Estrada. do Paço do Lumiar

1699 Lisboa Codex

PORTUGAL

Tel: $\quad+351(0) 1-7.16 .51 .41 / 7.16 .27 .12$

Fax: $\quad+351(0) 1-7.16 .09 .01$

Telex: $\quad 42486$ INETI P

E-mail: citi@ptearn.fc.ul.pt

02 Doctor Ana Maria Brigham da Silva Ramalho Correia (Director of CITI)

03 Academic staff: 11; one course administrator and one course secretary

04 in 1992/93: 26 students

05 Information Systems Development; Information Policy; Information Management

06 MSc in Information Management. Students' Handbook. Labaratório Nacional de Engenharia e Tecnologia Industrial, The University of Sheffield. [1992/93]

Curso de Mestrado em Gestão de Informação (Master of science (MSc) in Information Science) University of Sheffield, Department of Information Studies, Labaratório Nacional de Engenharia e Tecnologia Industrial, Centro de Informação Técnica para a Industria. 1993/94

Ramalho C., A. M.; Wilson, T. D.(1992): The MSc in Information Management of the University of Sheffield taught in Portugal: an example of knowledge transfer in education, in: Journal of Information Science 18, 1992, 77-82

07 Master of Science (MSc) in Information Management

! The Research Institute, in Association with the University of Sheffield, Department of Information Studies offers the MSc in Information Management through a combination of distance learning and local teaching. 


$\begin{array}{ll}\text { COUNTRY: } & \text { Portugal } \\ \text { CITY, INSTITUTION: } & \text { Lisbon, Universidade Católica Portuguesa } \\ \text { ACADEMIC SUBJECT: } & \end{array}$

01 Universidade Católica Portuguesa

Escola de Pós-Graduação

Campo da Palma de Cima

1600 Lisboa

PORTUGAL

Tel: $\quad+351(0) 1-7.26 .55 .50$

Fax: $\quad+351(0) 1-7.27 .02 .52$

02 Dr. Rodrigo de Magalhães

07 Information Management

! No more detailed information has been received recently.

$\begin{array}{ll}\text { COUNTRY: } & \text { Portugal } \\ \text { CITY, INSTITUTION: } & \text { Lisbon, Universidade de Lisboa } \\ \text { ACADEMIC SUBJECT: } & \end{array}$

01 Universidade de Lisboa

Faculdade de Letras

Alameda das Universidades

1699 Lisboa Codex

PORTUGAL

Tel: $\quad+351(0) 1-76.51 .62 / 77.96 .51 .67$

Fax: $\quad+351(0) 1-7.96 .00 .63$

02 Prof. Doctor Diago de Abreu

0320 enseignants à temps partiel

0420 étudiants

06 Reference of this item: International Council on Archives (1992): Répertoire des Ecoles et des cours de formation professionelle d'Archivistes/Directory of schools and courses of professional training for archivists. (Studies études, 6). Conseil International des Archives / International council on Archives. Koblenz 1992.

07 Certificat de spécialisation en sciences documentaires, Curso de especialização em ciencias documentais 
! L'établissement collabore notamment pour l'organisation des travaux pratiques avec les archives nationales, les archives historiques d'outre-mer, les archives bancaires, les archives parlementaires, les archives municipales de Lisbonne, les archives nationales de la photographie.

\section{COUNTRY: $\quad$ Romania \\ CITY, INSTITUTION: Bucureşti, Universitatea Bucureşti \\ ACADEMIC SUBJECT: Library and Information Science (p. 183)}

01 Universitatea Bucureşti

Facultatea de Litere

Sectia de Bibliologie si Stiinta Informárii

(Section de Bíbliologie et Sciences de l'Information)

Strada Edgar Quinet

Sector I, Cod 70778

Bucureşti

ROMÂNIA

Tel: $\quad+40(0) 1-6.14-35.08 / 190$

02 Dr. Ion Stoica (Tel: $+40(0)-1-6.15-42.40)$

04 nombre d'étudiants: 150

07 Diplômé en Bibliothéconomie et Sciences de l'Information

COUNTRY: $\quad$ Russia

CITY, INSTITUTION: Moskva, Inst. Povyšen. Kvalifik. Informac. Rabotn. (Institut for

ACADEMIC SUBJECT: Advanced Training of Information Specialists)

01 Inst. Povyšen. Kvalifik. Informac. Rabotn. (IPKIR)

(Institut for Advanced Training of Information Specialists)

Ul. Usieviča, 22

125315 Moskva

POCСИЯ / ROSSIJA

02 Prof. Ju. Uchin (Rektor)

! No more detailed information has been received recently. 
COUNTRY:

Russia

CITY, INSTITUTION:

Moskva, Kolleg der Informatik und Dokumentalistik im

ACADEMIC SUBJECT: Moskauer Haus des Wissens

01 Коледш Информатики и Документалистикий пгри Московском Доме Знаниц̆

(Kolleg der Informatik und Dokumentalistik im Moskauer Haus des Wissens) Mocвa (Moskva),

ул.Б.Галушина, 7 POCСИЯ / ROSSIJA

02 проф. БP. Серов(Prof WR Serv, Dekan); TH. Aнаньева(Dazinin T. N. Anajiewa)

07 Information management

! No more detailed information has been received recently.

COUNTRY:

Russia

CITY, INSTITUTION: Moskva, Moskovskij Gosudarstvennyi istoriko-archivnyj Institut

ACADEMIC SUBJECT:

01 Moskovskij Gosudarstvennyi istoriko-archivnyj Institut (MGIAI)

Ul. 25. Oktjabrja, 15

103642 Moskva

POССИЯ / ROSSIJA

02 M. V. Larin (Dozent)

! No more detailed information has been received recently. 


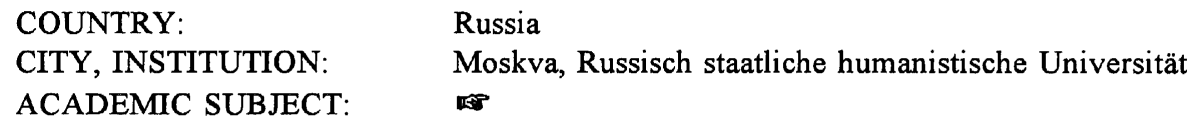

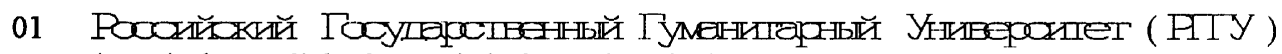
(Russisch staatliche humanistische Universität) 103642 Mосва (Moskva), Ул. Николъсая, 15 POCСИЯ / ROSSIJA

02 проф. А.Г. Романенко(Prof. A.G. Romanenko)

07 Informationswissenschaft

! No more detailed information has been received recently.

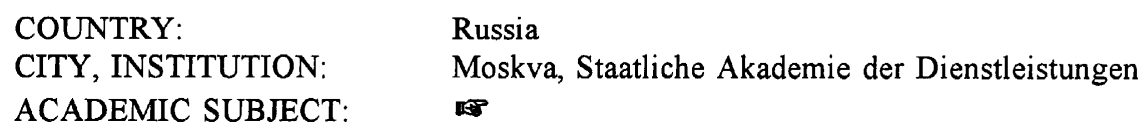

01 Госупарственная Акпемиа Сверы Быта и Услуг ( ГАСБУ) (Staatliche Akademie der Dienstleistungen)

141220 Mосковск. обл., Ст. Тарасовская улг. Главная 99 POCCИЯ / ROSSIJA

02 проф. Ю.П. Свирипенко(Prof. Ju.P. Swiridenko, Rektor)

07 Information management

! No more detailed information has been received recently. 


\section{COUNTRY:}

Russia

CITY, INSTITUTION:

Moskva, Moskauer Staatliches Kulturinstitut

ACADEMIC SUBJECT:

01 МБсковский Госупарственный Иенститут КултуУры ( МГИК) (Moskauer Staatliches Kulturinstitut)

141400 МоСКОВСК. Обл., Г. Химки- 6 (141400 Moskva oblast, Khimki 6) улл. Библиотечная 7. (ul. Bibliotechnaya 7)

POCСИЯ / ROSSIJA

Tel: $\quad+7(222)-570-31-33$

02 проф. Ю. Н. Столяров (Prof. Ju. N. Stolarow)

07 Information management / Informationswissenschaft

! No more detailed information has been received recently.

COUNTRY:

CITY, INSTITUTION:

ACADEMIC SUBJECT:

\section{Russia}

Petrograd, Petrograder N.K Krupskay Kulturinstitut

01 Petrograder N.K Krupskay Kulturinstitut

Dvortsovaya nab. 4

Petrograd D-41

POCCИЯ / ROSSIJA

Tel: $\quad+7(222)-312-95-12$

! No more detailed information has been received recently. 


$\begin{array}{ll}\text { COUNTRY: } & \text { Scotland } \\ \text { CITY, INSTITUTION: } & \text { Edinburgh, Queen Margaret College } \\ \text { ACADEMIC SUBJECT: } & \end{array}$

01 Queen Margaret College

Department of Communication and Information Studies

Edinburgh EH12 8TS

SCOTLAND

Tel: $\quad+41(0) 31-317-35.05$

Fax: $\quad+41(0) 31-316-41.65$

E-mail: cimmu@cis.queen-margaret-college.ac.uk / cimmu@uk.ac.qmced.cis (JANET)

02 George McMurdo (E-mail: geo_mcmurdo@hicom.org)

! No more detailed information has been received recently.

$\begin{array}{ll}\text { COUNTRY: } & \text { Slovakia } \\ \text { CITY, INSTITUTION: } & \text { Bratislava, Univerzita Komenského Bratislava } \\ \text { ACADEMIC SUBJECT: } & \end{array}$

01 Univerzita Komenského Bratislava

(Comenius University of Bratislava)

Filozofická Fakulta (Faculty of Arts)

Katedra knihovníctva a vedeckých informácií

(Department of Library and Information Science)

Gondova 2

81801 Bratislava

SLOVENSKO

Tel: $\quad+42(0) 7-32.36-08$

Fax: $\quad+42(0) 7-36.60 .16$

02 Assoc. Professor Štefan Kimlička (PhD)

03 Full-time staff: 5 associate professors/lecturers, 10 assistent professors, 3 scientific workers; part-time staff: $2-5$ teachers.

04 About 100 students

05 Analysis and design of librarying systems, retrospective cataloguing, training of Library and Information Science.

07 Master of Arts (MA), Doctor of Philosophy (PhD) in Library and Information Science 


\section{COUNTRY:}

CITY, INSTITUTION:

ACADEMIC SUBJECT:

\section{Spain \\ Barcelona, Escola Universitària Jordi Rubió i Balaguer \\ Library and Information Science (p. 184)}

01 Escola Universitària Jordi Rubió i Balaguer

de Biblioteconomia i Documentació

Travessera de les Corts

131-159 (Edifici Cambó)

08028 Barcelona

ESPAÑA

Tel: $\quad+34(9) 3-491-37.15$

Fax: $\quad+34(9) 3-491-39.54$

02 Mercè Bosch i Pou

06 Gui de l'alumne 1992-93 (1992): Escola Universitària Jordi Rubió i Balaguer de Biblioteconomia i Documentació. Diputació de Barcelona 1992

07 Diplomats Universitaris en Biblioteconomia i Documentació

$\begin{array}{ll}\text { COUNTRY: } & \text { Spain } \\ \text { CITY, INSTITUTION: } & \text { Barcelona, Universitat Autònoma de Barcelona } \\ \text { ACADEMIC SUBJECT: } & \quad \text { Information Science (p. 156) }\end{array}$

01 Universitat Autònoma de Barcelona

Facultat de Ciències de la Informació

Edifici I

08193 Bellaterra/Barcelona

ESPAÑA

Tel: $\quad+34(9) 3-581-19.45 / 19.83$

Fax: $\quad+35(9) 3-581-20.05$

Telex: 52040 EDUCIE

02 Enric Marín i Otto (Degà)

0357 professors i Departaments

$04 \quad 1981$ pla d'estudis

06 La guia de l'estudiant. 1992-1993. Universitat Autònoma de Barcelona, 1992

07 Llicenciatura en Ciències de la Informació 


\section{COUNTRY: $\quad$ Spain \\ CITY, INSTITUTION: Madrid, Centro de Información y Documentación Científica (CINDOC) \\ ACADEMIC SUBJECT: Information and Documentation (p. 126)}

01 Centro de Información y Documentación Científica

C.I.N.D.O.C (CSIC) antes ICYT

Calle Joaquín Costa 22

28002 Madrid

ESPAÑA

Tel: $\quad+34(9) 1-5.63-54.82$

Fax: $\quad+34(9) 1-5.64-26.44$

Telex: 22628 CIDMD E

E-mail: bibicyt@bib.csic.es

02 R. de la Viesca (Tel: +34(9)1-5.63-54.87)

0330 científicos ( 37 horas semanales)/80 no científicos ( 35 horas semanales)

05 Bibliometría, Terminología científica, Bases de datos bibliográficos, Biblioteconomía

07 Centrificado de en Información y Documentación

! Centro de investigación y servicios en ciencia y tecnología.

$\begin{array}{ll}\text { COUNTRY: } & \text { Spain } \\ \text { CITY, INSTITUTION: } & \text { Madrid, Universidad Autónoma de Madrid (UAM) } \\ \text { ACADEMIC SUBJECT: } & \quad \text { Information Science (p. 157) }\end{array}$

01 Universidad Autónoma de Madrid (UAM)

Facultad de Ciencias

Departamento de Química-Física Aplicada

Gabinete de Documentación Científica

Campus Canto Blanco

28049 Madrid

ESPAÑA

Tel: $\quad+34(9) 1-3.97 .47 .92$ (Secretaría)

Fax: $\quad+34(9) 1-3.97 .45 .12$

E-mail: sciendoc@vml.sdi.uam.es

02 Dra. Emilia Currás (Tel: $+34(0) 1-5.76 .97 .16=$ privado $)$

$03 \quad 2$ a 3 professores 
0430 a 40 estudiantes cado semestre

06 Currás, E. (1992): Glorias y miserias de los primeros años de SEDIC, Documentación de las Ciencias de la Información, 15, 1992, S. 131-147

07 Doctor en Ciencias de la Información

$\begin{array}{ll}\text { COUNTRY: } & \text { Spain } \\ \text { CITY, INSTITUTION: } & \text { Madrid, Universidad Carlos III de Madrid } \\ \text { ACADEMIC SUBJECT: } & \end{array}$

Madrid, Universidad Carlos III de Madrid

ACADEMIC SUBJECT

01 Universidad Carlos III de Madrid

Facultad Ciencias Sociales y Juridicales

Departamento de Biblioteconomía y Documentación

Calle Madrid 126-128

28903 Campus de Getafe (Madrid)

ESPAÑA

Tel: $\quad \quad \quad+34(9) 1-6.24 .99 .14 / 6.24 .98 .92$

Fax: $\quad+34(9) 1-6.24 .98 .91$

02 Dr. Mercedes Caridad Sebastian (Vice Dean, Tel: +34(9)1-6.24.95.43) / Dr. José Antonio Moreiro (Head of Department, Tel: +34(9)1-6.24.97.97)

031 Catedrático, 12 Titulares, 7 Ayudantes, 12 Asociados, 2 Administrativos

04550 en el Diplomatura; 30 en el Master

05 Nuevas Tecnologías, Automatización de Centros, Tratamiento y recuperación de la información

06 Universidad Carlos III de Madrid (1993): Facultad de Ciencias Sociales y Juridicales. Diplomatura en Biblioteconomía y Documentación. Plan de Estudios. 1993

07 Diplomado en Biblioteconomia y Documentacion Master en Informacion y Documentacion

! "Information is all around us [...] Without an uninterrupted flow of up-to-date and relevant information governments could not govern, industry commerce and the public services would cease to function and the everyday duties of citizenship, from abiding by the law to exercising the franchise, would become impossible" (William Martin, The Information Society, page 2). This quotation summarizes the philosophy behind Library and Information Studies at the Universidad Carlos III in Madrid. The Department of Library and Information Science opened in the academic year 1990/91 with the mandate to prepare information professionals capable of facing the challenges of the Information Age within the European Community. 
COUNTRY:

Spain

CITY, INSTITUTION:

Madrid, Universidad Complutense de Madrid

ACADEMIC SUBJECT:

Library Science (p. 206)

01 Universidad Complutense de Madrid

Escuela Universitaria de Biblioteconomía y Documentación

Ciudad Universitaria

28040 Madrid

ESPAÑA

Tel: $\quad+34(9) 1-5-49.02 .56$

Fax: $\quad+34(9) 1-5-3.94-34.37$

Telex: $\quad 41857$

02 Da. Concepcion Mendo Carmona

031 enseignant à plein temps, 2 archivistes professionels qui enseignent à temps partiel, 1 enseignant traite l'histoire et les sciences auxiliaires, 26 enseignants de la bibliothéconomie et des Sciences de l'information

04180 étudiants par an

06 Reference of this item: International Council on Archives (1992): Répertoire des Ecoles et des cours de formation professionelle d'Archivistes/Directory of schools and courses of professional training for archivists. (Studies études, 6). Conseil International des Archives/International council on Archives. Koblenz 1992.

07 Diplomatura en Biblioteconomía y Documentación

$\begin{array}{ll}\text { COUNTRY: } & \text { Spain } \\ \text { CITY, INSTITUTION: } & \text { Málaga, Universidad de Málaga } \\ \text { ACADEMIC SUBJECT: } & \end{array}$

01 Universidad de Málaga

Facultad de Ciencias de la Información

Campus El Ejido

Casa del Estudiante

290721 Málaga

ESPAÑA

Tel: $\quad+34(9) 52-13.10 .00$

Fax: $\quad+34(9) 52-26.38 .58$

! No more detailed information has been received recently. 


$\begin{array}{ll}\text { COUNTRY: } & \text { Spain } \\ \text { CITY, INSTITUTION: } & \text { Murcia, Universidad de Murcia } \\ \text { ACADEMIC SUBJECT: } & \end{array}$

01 Universidad de Murcia

Avda Teniente Flomesta s/n,

Edificio Convalecencia

30001 Murcia

ESPAÑA

Tel: $\quad+34(9) 68-21.72 .78$

Fax: $\quad+34(9) 68-22.15 .69$

Telex: $\quad 67058$

! No more detailed information has been received recently.

$\begin{array}{ll}\text { COUNTRY: } & \text { Spain } \\ \text { CITY, INSTITUTION: } & \text { Pamplona, Universidad de Navarra } \\ \text { ACADEMIC SUBJECT: } & \end{array}$

01 Universidad de Navarra

Instituto de Artes Liberales

31080 Pamplona

ESPAÑA

! No more detailed information has been received recently.

$\begin{array}{ll}\text { COUNTRY: } & \text { Spain } \\ \text { CITY, INSTITUTION: } & \text { Salamanca, Universidad de Salamanca } \\ \text { ACADEMIC SUBJECT: } & \text { Library Science (p. 207) }\end{array}$

01 Universidad de Salamanca

Escuela Universitaria de Biblioteconomía y Documentación

P. San Vincenta $s / \mathrm{n}$

37007 Salamanca

ESPAÑA

Tel: $\quad+34(9) 23-21.01 .34$

Fax: $\quad+34(9) 23-29.45 .02$

Telex: 294502 
02 M. Luis Hernandez Olivera

031 enseignant archiviste professionel, 4 enseignants traitant l'histoire et les sciences auxiliaires, 5 enseignants de la Bibliothéconomie et des Sciences de l'Information, 2 du Droit et de l'Administration

04225 étudiants

06 Reference of this item: International Council on Archives (1992): Répertoire des Ecoles et des cours de formation professionelle d'Archivistes/Directory of schools and courses of professional training for archivists. (Studies études, 6). Conseil International des Archives/International council on Archives. Koblenz 1992.

07 Diplomado en Biblioteconomía y Documentación

$\begin{array}{ll}\text { COUNTRY: } & \text { Sweden } \\ \text { CITY, INSTITUTION: } & \text { Borås, Högskolan i Borås (Swedish School of Library \& } \\ & \text { Information Science) }\end{array}$

ACADEMIC SUBJECT: Library and Information Science (p. 186)

01 Postal address

Visiting address:

Högskolan i Borås

Swedish School of Library \& Information Science (SSLIS)

Box 874

Allégatan 1

50115 Borås

50115 Borås

SVERIGE

SVERIGE

Tel: $\quad+46(0) 33-16.40 .00$

Fax: $\quad+46(0) 33-11.95 .98$

02 Romulo Enmark (Head of Department) / Chagan Lalloo (Director of Studies)

03 Administrative staff: 8; faculty: full-time 19; part-time, guests: 80-100 (FTE-2,5)

04480 Students

05 The general aims of the Centre are to monitor and indirectly coordinate, library and information-related research in Sweden; engage in, promote and encourage research in the field and finally, work towards the establishment of a doctoral programme in Librarianship and Information Studies.

06 Chagan, L. (1990): Swedish School of Library and Information Science. A Presentation. Bibliotekshögskolan Borås 1990

Studying in Sweden. Higher Education for Visiting Students. Admission to the Preparatory Swedish Language Course and Undergraduate Studies. Admission to Postgraduate Studies (1991): National Board of Universities and Colleges, Department for Student Affairs, Stockholm 1991 
07 Master in Library and Information Science

! The maintenance costs in Sweden are high (about SEK 5.000 per month). No scholarships or other fincancial assistance are available from the Swedish School of Library and Information Science, foreign students are permitted to work during the summer vacations but not during semester time.

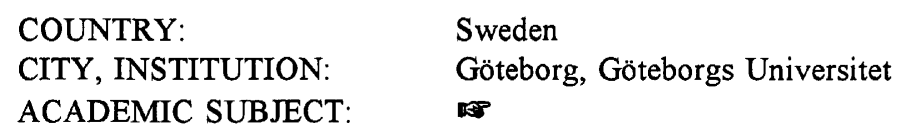

01 Göteborgs Universitet

Centrum för biblioteks- och informationsvetenshap

Brogatan 4

41301 Göteborg

SVERIGE

Fax: $\quad+46(0) 31-7.73 .49 .33$

E-Mail: brigitta.barankop@ctv.gu.se

02 Erland Munch-Petersen (Acting professor)

! No more detailed information has been received recently.

$\begin{array}{ll}\text { COUNTRY: } & \text { Sweden } \\ \text { CITY, INSTITUTION: } & \text { Linköping, Universitetet i Linköping } \\ \text { ACADEMIC SUBJECT: } & \end{array}$

01 Universitetet i Linköping

Department for Education and Psychology

58183 Linköping

22100 Lund

SVERIGE

Tel: $\quad+46(0) 13-28.10 .00$

Fax: $\quad+46(0) 13-28.21 .45$

02 Maj Klasson (Associate Professor)

! No more detailed information has been received recently. 


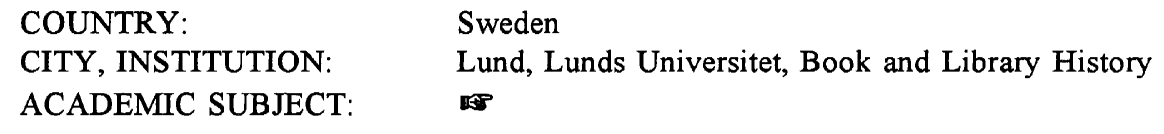

ACADEMIC SUBJECT:

01 Lunds Universitet

Book und Library History

POB 117

22100 Lund

SVERIGE

Tel: $\quad+46(0) 46-10.70 .00$

Fax: $\quad+46(0) 46-10.40 .20$

02 Professor Per S. Ridderstad

! No more detailed information has been received recently.

$\begin{array}{ll}\text { COUNTRY: } & \text { Sweden } \\ \text { CITY, INSTITUTION: } & \text { Lund, Lunds Universitet, Informationsbehandling-ADB } \\ \text { ACADEMIC SUBJECT: } & \infty \quad \text { Information and Computer Science (p. 118) }\end{array}$

01 Lunds Universitet

Informationsbehandling-ADB

Sölvegatan 14A

22362 Lund

SVERIGE

Tel: $\quad+46(0) 46-10.70 .00$

Fax: $\quad+46(0) 46-10.45 .28$

E-mail: ibadb@ibadb.lu.se / Kjell-Ake.Holmberg@ibadb.lu.se

02 Dipak Khakhar (Head of Department, Tel: +46(0)46-10.80.28)/ Kjell-Åke Holmberg (Director of Studies, Tel: 046-10.73.38)

036 Doctors of Philosophy (one of them as Professor); 18 teachers; 3 non-scientific employees

04 About 800 students.

06 Systems Development, Systems Design, Prototyping, User Development

06 Gerbro-Bülow, E. / Khakhar, D. (1992): Lund Universitet. Information and Computer Science. Lund 1992

Studying in Sweden. Higher Education for Visiting Students. Admission to the Preparatory Swedish Language Course and Undergraduate Studies. Admission to Postgraduate 
78

Studies (1991): National Board of Universities and Colleges, Department for Student Affairs, Stockholm 1991

07 Bachelor of Science (BA), Master of Social Science (MSSC), Doctor of Philosophy $(\mathrm{PhD})$ in Information and Computer Science

$\begin{array}{ll}\text { COUNTRY: } & \text { Sweden } \\ \text { CITY, INSTITUTION: } & \text { Umeå, Umeå Universitet } \\ \text { ACADEMIC SUBJECT: } & \text { U }\end{array}$

01 Umeå Universitet

Department of Media and Communication

90187 Umeå

SVERIGE

Tel: $\quad+46(0) 90-16.50 .00$

Fax: $\quad+46(0) 90-16.54 .88$

Telex: $\quad 54005$

02 Professor Lars Hglund

! No more detailed information has been received recently.

$\begin{array}{ll}\text { COUNTRY: } & \text { Switzerland } \\ \text { CITY, INSTITUTION: } & \text { Genève, Ecole Supérieure d' Information Documentaire } \\ \text { ACADEMIC SUBJECT: } & \end{array}$

01 Ecole Supérieure d'Information Documentaire Institut d'Etudes Sociales

28 rue Prévost-Martin

1211 Genève 4

SUISSE

Tel: $\quad+41(0) 22-3.20-93.11$

Fax: $\quad+41(0) 22-3.20-72.46$

02 Yolande Estermann-Wiskott (Responsable d'Ecole)

03 Equipe permanente: 5,1 postes pédagogiques. Généralement à $3 / 4$ ou plein temps $+2,5$ secrétaires. Enseignants extérieurs: ne viennent que pour enseigner (entre $4 \mathrm{~h}$ et $40 \mathrm{~h}$ par an).

0475 étudiants 
06 Plan de formation et Conditions d'admission (1993): Ecole Supérieure d'Information Documentaire. Genève 1993

07 Diplôme en Bibliothéconomie, Documentation et Archivistique

! En collaboration avec l'E.S.I.D. la Faculté des Lettres de l'Université de Genève propose une formation postgraduates en information documentaire: Certificat de spécialisation en Information Documentaire (CESID).

COUNTRY:

CITY, INSTITUTION:

ACADEMIC SUBJECT:
Switzerland

St. Gallen, Hochschule

Information Management (p. 138)

01 Hochschule St. Gallen Institut für Wirtschaftsinformatik (IWI)

Dufourstr. 50

9000 St. Gallen

SCHWEIZ

Tel: $\quad+41(0) 71-30.24 .20$

Fax: $\quad+41(0) 71-22.87 .56$

E-mail: oesterle@csghsg5a.bitnet / mderungs@sgcll.unisg.ch

02 Secretariate of the university / Prof. Dr. H. Oesterle (Director of the Institute of Information Management)

04 About 30 students p.a.

05 The Institute of Information Management has set up the research programme "Information Management 2000" in which 17 major companies from Switzerland and Germany are conducting research with the Institute of Information Management. Our research is organized in five competence centres with following principle fields of research: Controlling and Redesigning of Information Systems, Computer-based Information Management, Electronic Markets, Electronic Information Assistant and TeleCounter.

06 Studienpläne und Prüfungsordnung. Grundstufe (1991): Hochschule St. Gallen. 1991 Vorlesungsverzeichnis Wintersemester 1992/93 (1992): Hochschule St. Gallen. 1992 Informations- und Technologiemanagement an der HSG. Hochschule St. Gallen[o.J.] ITEM-HSG, Institut für Technologiemanagamen (1993): Vertiefungsrichtung Technologiemanagement. Hochschule St. Gallen. 1993

IWI, Institut für Wirtschaftsinformatik (1993): Vertiefungsrichtung Informationsmanagment. Hochschule St. Gallen 1993

IfU, Institut für Unternehmensforschung (Operations Research) (1993): Studienrichtung Informations- und Technologiemanagment. Hochschule St. Gallen 1993 
07 Lizenziat der Wirtschaftswissenschaften der Hochschule Sankt Gallen (lic. oec. HSG), Doktor der Wirtschaftswissenschaften Sankt Gallen (Dr. oec. HSG) in Information and Technology Management.

$\begin{array}{ll}\text { COUNTRY: } & \text { Ukraine } \\ \text { CITY, INSTITUTION: } & \text { Kiev, Kiev State Institute of Culture } \\ \text { ACADEMIC SUBJECT: } & \end{array}$

01 Kiev State Institute of Culture

Department of Library and Information Systems

Ul. Shchorsa 36

Kiev

UKRAIJNA

Tel: $\quad+7(222)-295-44-03$

! No more detailed information has been received recently.

$\begin{array}{ll}\text { COUNTRY: } & \text { United Kingdom } \\ \text { CITY, INSTITUTION: } & \text { Aberdeen, Robert Gordon University } \\ \text { ACADEMIC SUBJECT: } & \text { Information Analysis (p. 113) } \\ & \text { Library and Information Studies (p. 187-188) }\end{array}$

01 Robert Gordon University

School of Librarianship and Information Science

Hilton Place

Aberdeen AB9 1FP

UNITED KINGDOM

Tel: $\quad+44(0) 224-28.38 .30 .0$

Fax: $\quad+44(0) 224-22.44 .88 .54 .5$

E-mail: slis@rgu.ac.uk

02 Ian M. Johanson (Head of School, Tel: +44(0)224-28.38.37)

$031 \mathrm{Head}$ of School, 3 Senior Lecturers, 8 Lecturers (full-time), 1 Lecturer (part-time), 1 Computer Technician, 1 Secretary

04 About 150 full-time students and about 50 part-time students at school.

05 All aspects of information. 
07 . Bachelor of Arts (BA (Hons)), Master of Arts (MA), Doctor of Philosophie (PhD) in Library and Information Studies

Postgraduate Diploma (PgD), Master of Arts (MA), Master of Science (MSc) in Library and Information Studies

Postgraduate Diploma (Pg), Master of Science (MSc) in Information Analysis

COUNTRY:

CITY, INSTITUTION:

ACADEMIC SUBJECT:
United Kingdom

Aberystwyth, University of Wales

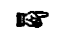

01 University of Wales

Department of Information an Library Studies

Llanbadarn Fawr

Aberystwyth, Dyfed SY23 3AS

UNITED KINGDOM

Tel: $\quad+44(0) 970-62.21 .88$

Fax: $\quad+44(0) 970-62.21 .90$

E-Mail: dils@aber.ac.uk

02 Hywel E. Roberts (Head of Department)

0325 academic staff

04500 students

05 Library and Information work in a welsh context, Information Management, Information Retrieval, Social Histroy of Libraries, Health and Welfare Information Management.

07 Bachelor of Science (BSc) in Information Science

Bachelor of Science (BSc) in Information and Library Studies Master of Arts (MA) in Information and Library Studies

Master of Science (MSc) in Health Information Management

! The Department is the largest centre in the UK teaching and researching in the areas of Information Science and Librarianship.

No more detailed information has been received recently. 
COUNTRY:

CITY, INSTITUTION:

ACADEMIC SUBJECT:
United Kingdom

Birmingham, University of Central England (UCE) in Birmingham

Information and Library Studies (p. 129)

Librarianship and Information Studies (p. 189)

01 University of Central England (UCE) in Birmingham

Faculty of Computing and Information Studies

School of Information Studies

Perry Barr

Birmingham B42 2SU

UNITED KINGDOM

Tel: $\quad+44(0) 21-3.31-56.25$

Fax: $\quad+44(0) 21-3.31-56.81$

E-mail: j.elkin@uce.ac.uk

02 Prof. Judith Elkin (Head of School, Tel: +44(0)21-3.31-56.25)

$03 \quad 15$ academic staff; 2 research staff

04240 students

05 Information Networks; School Libraries; Children's Literature; Multicultural Studies; Local Studies; European Information

06 BA (Hons) Information and Library Studies (1990): Definitive Document. Faculty of Computing and Information Studies. School of Information Studies. Birmingham 1990 Postgraduate Diploma/MA/MSc in Librarianship and Information Studies (1989): Definitive Document. Birmingham Polytechnic. Faculty of Computing and Information Studies. Department of Librarianship and Information Studies. Birmingham 1989

07 Bachelor of Arts (BA (Hons)) in Information and Library Studies Postgraduate Diploma (PgD), Master of Arts (MA), Master of Science (MSc), Doctor of Philosophy $(\mathrm{PhD})$ in Librarianship and Information Studies 
COUNTRY:

CITY, INSTITUTION:

ACADEMIC SUBJECT:
United Kingdom

Bradford, University of Bradford

Computing and Information Systems (p. 103)

01 University of Bradford

Department of Computing

Phoenix Building

Bradford BD7 1DP

UNITED KINGDOM

Tel: $\quad+44(0) 2.74-38.39 .51$

Fax: $\quad+44(0) 2.74-383920$

E-mail: j.stephenson@comp.brad.ac.uk

02 Prof. John Stephenson (Head of Department, Tel. $+44(0) 2.74-38.39 .51$ )

0319 academic teaching/research staff; 2 part-time teaching staff; 9 technical support staff; 4 secretarial support staff (all full-time)

04 About 250 full-time students in the Department of Computing.

05 Databases (conventional and Intelligent Knowledge Based Systems); Library records; Wide range of applications

07 Bachelor of Science (BA (Hons)) in Computing and Information Systems

COUNTRY:

CITY, INSTITUTION:

ACADEMIC SUBJECT:

\author{
United Kingdom \\ Brighton, University of Brighton \\ Computing and Information Systems (p. 104) \\ Information Systems (p. 166) \\ Library and Information Studies (p. 190)
}

01 University of Brighton

Faculty of Information Technology

Moulescoomb

Brighton BN2 4G3

UNITED KINGDOM

Tel: $\quad+44(0) 2.73-60.09 .00$

Fax: $\quad+44(0) 2.73-64.24 .05$

02 Prof. Gordon M. Bull (Dean of Faculty) / Prof. Dan Simpson (Head of Department: Computing), Peter Enser (Head of Department Library and Information Studies) / Sandra Landy (course leader Computing and Information Systems, Tel: +44(0)273-64.24.70) / 
Duncan Quibell (course leader Information Systems, Tel: +44(0)273-64.24.69) / Jim Hennessy (course leader Library and Information Studies, Tel: +44(0)273-64.35.00)

04200 students on the degree Computing and Information Systems, 600 students in department and 8000 in university.

05 This Faculty of Information Technology is at the forefront of teaching and research in the general area of Information Technology. We have a substantial research and consultancy activity in all departments with major research projects and some twenty research students based in the IT Research Institute.

06 First degree diploma \& certificate full-time \& sandwich courses (1993): 1993 entry. University of Brighton 1993

Computing and Information Systems BA(Hons) (1991): Faculty of Information Technology, Department of Computing. Brighton Polytechnic 1991

MSc Information Systems. Course Outline (1991): Faculty of Information Technology, Department of Computing. Brighton Polytechnic 1991

07 Bachelor of Science (BSc), Diploma of Higher Education (DiphHE) in Computing and Information Systems

Master of Science (MSc), Postgraduate Diploma (PgD) in Information Systems

Bachelor of Arts (BA (Hons)), Master of Arts (MA) in Library and Information Studies

! We offer Higher National Diplomas in Computing and Mathematical Studies, Honours Degrees in Computer Science, Computing and Information Systems, Mathematics for Management, Library and Information Studies and a wide ranging group of degrees under the banner of the Modular Science Scheme, and Masters Degrees in Information Systems, Information Studies and Software Engineering. One of the courses we offer is a joint courses with Paris which enables those with a good HND in computing to study for one year, half in Brighton and half in Paris to obtain a UK degree from Brighton and a Diplôme d'Etudes Supérieures Technologiques from the IUT de l'Université de Paris V. 
COUNTRY:

CITY, INSTITUTION:

ACADEMIC SUBJECT:

\author{
United Kingdom \\ Glasgow, University of Strathclyde \\ Information and Library Sciences (p. 130) \\ Information Management (p. 139) \\ 10 Information Science (p. 158)
}

01 University of Strathclyde

Department of Information Science

Strathclyde Business School

Glasgow GI IXN

UNITED KINGDOM

Tel: $\quad+41(0) 552-44.00 / 37.00$

Fax: $\quad+41(0) 553-13.93$

E-mail: secretary@dis.strath.ac.uk

02 Prof. Charles Oppenheim (Head of Department) / Paul F. Burton (Course Leader I.L.S)/ Crawford Revie (Course Leader I.M.) / Douglas Badenoch (Course Leader I.S.)

0312 academic staff; 12 scientific employees, 2 technican staff

0450 students in Information and Library Studies; 35 in Information Management, 70 in Information Science

05 Information Retrieval, Business Information Systems

06 Cronin, B. (1989): University of Strathclyde, Glasgow, UK, in: Education for Information, $1989,7,157-162$

07 Master of Arts (MA), Postgraduate Diploma (PgD) in Information and Library Studies Master of Science (MSc), Postgraduate Diploma (PgD) in Information Management Master of Science (MSc), Diploma, Honours Degree of Equivalent in Information Science 
COUNTRY:

CITY, INSTITUTION:

ACADEMIC SUBJECT:
United Kingdom

Leeds, Leeds Metropolitan University

US?

01 Leeds Metropolitan University

Faculty of Information \& Engineering Systems

Information and Library Studies Group

Beckett Park

Leeds LS6 3QS

UNITED KINGDOM

Tel: $\quad+44(0) 532-83.26 .00$

Fax: $\quad+44(0) 532-83.31 .82$

E-Mail: b.livesey@lmu.ac.uk

02 Brian Livesey (Group Leader)

$03 \quad 14$ academic staff

$04 \quad 252$ students

05 Community Librarianship; Library History; Image Retrieval; Personnel Management; Information Services for young people; Cataloguing; Library Automation and Text Processing Systems.

07 Bachelor of Arts (BA (hons)) in Information Studies

Master of Arts (MA) in Librarianship

Master of Science (MSc) in Information Studies

Postgaduate Diploma (PgD) in Information Studies

! No more detailed information has been received recently.

COUNTRY:

CITY, INSTITUTION:

ACADEMIC SUBJECT:

\section{United Kingdom}

Liverpool, Liverpool John Moores University

Information and Library Studies (p. 131)

01 Liverpool John Moores University

Liverpool Business School

98 Mount Pleasant

Liverpool L3 5UZ

UNITED KINGDOM

Tel: $\quad+44(0) 51-2.31-21.21$

Fax: $\quad+44(0) 51-7.07-04.23$

E-Mail: list@livjm.ac.uk 
02 Prof. Denis Smith (Director of School) / Eric J. Hunter (Subject Head, Tel: +44(0)512.31-35.89)

039 full-time teaching staff in Information and Library Studies plus servicing from other groups. General administrative/ technician support from the Liverpool Business School.

04 Ca. 200 full-time, ca. 30 part-time students

05 Liaison with a number of outside institutions and organizations.

07 Bachelor of Arts (BA (Hons)) in Information and Library Studies Bachelor of Sciences (BSc (Hons)) in Information and Library Studies Diploma, Master of Arts (MA) in Information and Library Studies Master of Philosophy (MPhil) in Information and Library Studies

COUNTRY: $\quad$ United Kingdom

CITY, INSTITUTION: London, City University of London

ACADEMIC SUBJECT: Information Science (p. 159)

Health Informatics (p. 113)

01 City University of London

Department of Information Science

Northampton Square

London, EC1V OHB

UNITED KINGDOM

Tel: $\quad+44(0) 71-477-83.80$

Fax: $\quad+44(0) 71-477-85.84$

E-mail: dab@is.city.ac.uk / slb@is.city.ac.uk

02 Prof. S.E. Robertson (Head of Department) / David Bawden (Course Director)

037 full-time, 2 part-time academic staff; numerous visiting lecturers; 4 administrative staff

0440 students

05 Interactive Systems; Medical Informatics; Theories of Information, Information flow and dissemination

06 Rennie, J. S. (1986): A history of the Department of Information Science of the City University, in: Journal of Information Science 12, 1986, 3-13

07 Master of Science (MSc), Diploma, postgraduate Diploma in Information Science Bachelor of Science (BSc) in Health Informatics 


\title{
COUNTRY: United Kingdom \\ CITY, INSTITUTION: London, Thames Valley University \\ ACADEMIC SUBJECT:

01 Thames Valley University

Faculty of Business and Information Studies

Department of Information Studies and Technology

St. Mary's Road, Ealing

London, W5 5RF

UNITED KINGDOM

Tel: $\quad+44(0) 81-5.79-50.00$

! Founded 1991; formerly Ealing College of Higher Education.

No more detailed information has been received recently.

\author{
COUNTRY: $\quad$ United Kingdom \\ CITY, INSTITUTION: London, University College of London \\ ACADEMIC SUBJECT: Archive Studies (p. 97) \\ Information Science: Computerized Systems (p. 159) \\ Library and Information Studies (p. 191)
}

01 University College of London

School of Library, Archive and Information Studies

Gower Street

London WCIE 6BT

UNITED KINGDOM

Tel: $\quad+44(0) 71-3.87-70.50 / 72.04$

Fax: $\quad+44(0) 71-3.82-05.57$

02 Prof. Robert Alston (Director); Mrs. Gillian Martin (Lecture i/c MSc course, Tel: $+44(0) 71-3.87-70.50-72.04)$

039 persons teaching staff; 2 persons Department Office; 1 person technical staff;

0420 students

06 MSc Students' Handbook 1992-1993 (1992): A guide for students taking the MSc (Information Science). Computerized Systems for Librarians, Archivists and Information Managers. School of Library, Archive \& Information Studies. University College London. September 1992

School of Library, Archive and Information Studies. Issued 1992. Dates of Terms (1992): Session 1992-1993. 1992 
07 Master of Arts (MA), Diploma, Master of Philosophie (MPhil), Doctor of Philosophy $(\mathrm{PhD})$ in Archive Studies

Master of Science (MSc) in Information Science: Computerized Systems; Library and Information Studies

COUNTRY:

CITY, INSTITUTION:

ACADEMIC SUBJECT:

\author{
United Kingdom \\ London, University of North London, Faculty ... \\ Information and Communication (p. 116)
}

01 University of North London

Faculty of Environmental and Social Studies

Ladbroke House 62-66

Highbury Grove

London N5 $2 \mathrm{AD}$

UNITED KINGDOM

Tel: $\quad+44(0) 71-753-50.31$

Fax: $\quad+44(0) 71-753-51.00$

Telex: 25228 PNL G

02 A.J. Beard (Senior Lecturer)

03 The Faculty of Environmental and Social Studies has 170 staff of which 130 teach on the ASS Modular Degree.

041700 students

07 Bachelor of Science (BSc (Hons)) in Applied Social Sciences (Information and Communication)

! We are pleased to welcome pre-arranged visits to the Faculty and try to provide staff to talk to you about the courses and advise on preparatory study, possible exemptions, etc. We provide staff at the regular drop-in advice sessions run by PNL at the Holloway Road site on the first Thursday of every month as well organizing our own Open Day and advisory sessions. 


\section{COUNTRY: $\quad$ United Kingdom \\ CITY, INSTITUTION: $\quad$ London, University of North London, School ... \\ ACADEMIC SUBJECT: Information Studies (p. 162)}

01 University of North London

School of Information Studies

Ladebroke House

62-66, Highsbury Grove

London N5 2AD

UNITED KINGDOM

Tel: $\quad+44(0) 71-607-27.89$

Fax: $\quad+44(0) 71-753-51.00$

Telex: 25228 PNL G

02 Mrs. R. Raddon (Head of School)

$03 \quad 12$ full-time and 6 part-time staff

04180 undergraduates and 170 postgraduates

05 User services; Online systems; Intelligent Systems; Hypermedia

07 Master of Arts (MA), postgraduate Diploma in Information Studies

COUNTRY:

ACADEMIC SUBJECT: Information and Computing Studies (p. 119)

Information Studies (p. 164)

Records Management \& Archives (p. 98)

01 Loughborough University of Technology

Department of Information and Library Studies

Leicestershire LE11 3TU

UNITED KINGDOM

Tel: $\quad+44(0) 509-22.30 .52$

Fax: $\quad+44(0) 509-22.30 .53$

E-mail: dils@lut.ac.uk / j.p.feather@lut.ac.uk

02 John P. Feather (Head of Department, Tel: +44(0)509-22.30.50) / Mrs. Hilary Dyer (Senior Lecturer, Tel: +44(0)509-22.30.68) 
0322 academic staff; 9 administration; 2 research fellows; 3 research assistants; 3 library staff

04 Ca. 3000 Students

05 The department gets research grants in 1991/92 in: Study of Publisher's Databases; Strategic Management of Public Library Services; Information needs of the travelling public; Marketing of Public Libraries and Leisure Services in the U.K.; Comparision of value of documents retrieved by end-user searching for CD-ROM based index services; Computers in Teaching Initiative; Supports to Learning; Humanities Information Review Panel; Expert Systems for On-Line Database Selection; Library \& Information Statistics Unit; To organise a drafting meeting and launch for the report of the humanities Review Panel; Policy Making and Strategic Management course.

06 Undergraduate Prospectus October 1993 Entry (1992). Loughborough University of Technology 1992

Postgraduate Courses and Research. 1992/93 (1992): Loughborough University of Technology 1992

07 Bachelor of Science (BSc (Hons) in Information and Computing Studies Bachelor of Arts (BA), Bachelor of Science (BSc (Hons) in Information and Library Studies

Master of Arts (MA) Master of Science, Diploma of Loughborough University of Technology (DLUT) in Information and Library Studies

Master of Arts (MA), Master of Science (MSc), Diploma of Loughborough University of Technology (DLUT) in Information Studies

Master of Arts (MA), Master of Science (MSc), Diploma of Loughborough University of Technology (DLUT) in Records Management \& Archives

\section{COUNTRY:}

CITY, INSTITUTION: ACADEMIC SUBJECT:

\author{
United Kingdom \\ Manchester, Manchester Metropolitan University \\ 10 Information and Library Management (p. 126) \\ Information Management (p. 139) \\ Strategic Library Management (p. 209)
}

01 Manchester Metropolitan University

Department of Library and Information Studies

All Saints

Manchester M15 6BH

UNITED KINGDOM

Tel:

$+44(0) 61-247-61.44$ 
02 Mr. P.W. Lea (Course Leader Information and Library Management) / Jonathan Willson (Course Leader Information Management (postgraduate)) / David Kaye (Course Leader Strategic Library Management)

$03 \quad 16$ full-time lecturers; 7 support staff

04200 full/part-time students

05 OPAC; Hypertext; Management

07 Bachelor of Arts (BA (Hons)) in Information and Library Management Master of Science (MSc) in Information Management Master of Arts MA) in Strategic Library Management

COUNTRY:

CITY, INSTITUTION: ACADEMIC SUBJECT:

\author{
United Kingdom \\ Newcastle upon Tyne, University of Northumbria at Newcastle \\ Information and Library Management (p. 128) \\ Learning Resource Centre Management (p. 192)
}

01 University of Northumbria at Newcastle

Department of Information and Library Management

Lipman Building

Newcastle upon Tyne NE1 8ST

UNITED KINGDOM

Tel: $\quad+44(0) 91-2.35-89.17$

Fax: $\quad+44(0) 91-2.35-85.72$

E-mail: liv3@vaxa.npy.ac.uk

02 Mrs. Joan, M. Day (Tel: +44(0)-91-2.35-37.02)

0316 full-time academic staff; 1,5 secretarial support staff; 1 technician

04220 students in department

05 The study of information service use, and communities of users and potential users associated with such use. The study of Information Technology as a device for supporting information services and the systems accommodating those services.

06 Kemp, D. A. (1989): Newcastle upon Tyne Polytechnic: End Exercises, in: Education for Information, 1989, 7, 159-161 
07 Bachelor of Arts (BA) in Information and Library Management, University Diploma, Master of Arts (MA), Master of Science (MSc), Master of Philosophy (MPhil), Doctor of Philosophy (PhD)

Diploma in Learning Resource Centre Management

! All units of all courses are credited to allow transfer between European institutions.

\section{COUNTRY: CITY, INSTITUTION: ACADEMIC SUBJECT:}

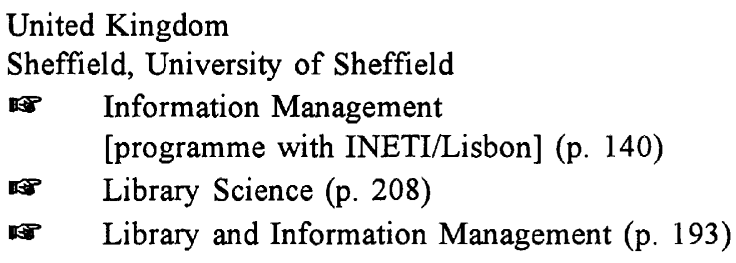

01 Postal address:

Visiting address:

University of Sheffield

Department of Information Studies

Western Bank

Sheffield S10 2TN

UNITED KINGDOM
Regent Court, 211 Portobello

Sheffield, S10 2SE

UNITED KINGDOM

Tel: $\quad+44(0) 7.42-82.50 .81$

Fax: $\quad+44(0) 7.42-78.03 .00$

Telex: $\quad 547216$ UGSHEF G

E-mail: $\quad$ t.d.wilson.sheffield.ac.uk

02 Tom Wilson (Head of Department) / Dr. Ana M. B. da S. R. Correia (programme coordinator for the MSc in Information Management in Lisbon)

035 professors; 7 full-time lecturers; 4 part-time lecturers; 7 staff for the MSc programme in Lisbon; 3 non academic staff; 6 additional to academic staff

05 The department attracts many research students from outside Britain. Current research in the department includes Studies of Information needs of many groups, including managers in business and local authority departments; the impact of Information Technology in organizations, Public Policy and Library and Information Services, academic and public Library Management, computer-based Information Storage and retrieval systems, Automatic Text Processing, searching algorithmens, representation and searching of three-dimensional chemical structures and of generic structures in patents, chemical applications of graph theory and non-numeric applications of parallel computer hardware. 
06 Saunders, W. L. (1989): The University of Sheffield Department of Information Studies, 1964-1989, in: Journal of Information Science 15, 1989, 193-202

MSc in Information Management. Students' Handbook (1992/1993): Labaratório Nacional de Engenharia e Tecnologia Industrial, The University of Sheffield. [1992/93]

Curso de Mestrado em Gestão de Informação (Master of Science (MSc) in Information Science) (1993/94): University of Sheffield, Department of Information Studies, Labaratório Nacional de Engenharia e Tecnologia Industrial, Centro de Informação Técnica para a Industria. 1993/94

Ramalho C., A. M. / Wilson, T. D. (1992): The MSC in Information Management of the University of Sheffield taught in Portugal: an example of knowledge transfer in education, in: Journal of Information Science 18, 1992, 77-82

07 Master of Science (MSc) in Information Management

Master of Science (MSc) in Information Management (INETI/Lisbon)

Master of Arts (MA) in Librarianship

Master of Arts (MA) in Library and Information Management

Master of Business Administration (MBA) Information Management option

Bachelor of Science (BSc) in Business Studies and Information Management (start from the academic year 1993/94)

Bachelor of Science (BSC) in Financial Management \& Accounting and Information Management (start from the academic year 1993/94)

! The Department, in Association with the Labaratorio National de Engenharia e Tecnologia Industrial (INETI), offers the MSc in Information Management through a combinition of distance learning and local teaching on the campus of INETI at Lumiar, Lisbon, Portugal.

The fees for full-time postgraduate students are subject to review peridiocally as the University reserves the right to alter fees at any time. Fees for individual programmes are available on application.

The Department welcomes applications from suitably qualified candidates wishing to undertake research for an MPhil, or PhD by thesis. 